\title{
Chiral approach to nuclear matter: Role of two-pion exchange with virtual delta-isobar excitation ${ }^{1}$
}

\author{
S. Fritsch, N. Kaiser, and W. Weise \\ Physik-Department, Technische Universität München, D-85747 Garching, Germany \\ email: nkaiser@physik.tu-muenchen.de
}

\begin{abstract}
We extend a recent three-loop calculation of nuclear matter in chiral perturbation theory by including the effects from two-pion exchange with single and double virtual $\Delta$ (1232)-isobar excitation. Regularization dependent short-range contributions from pionloops are encoded in a few NN-contact coupling constants. The empirical saturation point of isospin-symmetric nuclear matter, $\bar{E}_{0}=-16 \mathrm{MeV}, \rho_{0}=0.16 \mathrm{fm}^{-3}$, can be well reproduced by adjusting the strength of a two-body term linear in density (and tuning an emerging three-body term quadratic in density). The nuclear matter compressibility comes out as $K=304 \mathrm{MeV}$. The real single-particle potential $U\left(p, k_{f 0}\right)$ is substantially improved by the inclusion of the chiral $\pi N \Delta$-dynamics: it grows now monotonically with the nucleon momentum $p$. The effective nucleon mass at the Fermi surface takes on a realistic value of $M^{*}\left(k_{f 0}\right)=0.88 M$. As a consequence of these features, the critical temperature of the liquid-gas phase transition gets lowered to the value $T_{c} \simeq 15 \mathrm{MeV}$. In this work we continue the complex-valued single-particle potential $U\left(p, k_{f}\right)+i W\left(p, k_{f}\right)$ into the region above the Fermi surface $p>k_{f}$. The effects of $2 \pi$-exchange with virtual $\Delta$-excitation on the nuclear energy density functional are also investigated. The effective nucleon mass associated with the kinetic energy density is $\widetilde{M}^{*}\left(\rho_{0}\right)=0.64 M$. Furthermore, we find that the isospin properties of nuclear matter get significantly improved by including the chiral $\pi N \Delta$-dynamics. Instead of bending downward above $\rho_{0}$ as in previous calculations, the energy per particle of pure neutron matter $\bar{E}_{n}\left(k_{n}\right)$ and the asymmetry energy $A\left(k_{f}\right)$ now grow monotonically with density. In the density regime $\rho=2 \rho_{n}<0.2 \mathrm{fm}^{-3}$ relevant for conventional nuclear physics our results agree well with sophisticated many-body calculations and (semi)-empirical values.
\end{abstract}

PACS: 12.38.Bx, 21.65.+f, 24.10.Cn, 31.15.Ew

Keyword: Nuclear matter properties; Two-pion exchange with virtual $\Delta(1232)$-isobar excitation

\section{Introduction and preparation}

In recent years a novel approach to the nuclear matter problem based on effective field theory (in particular chiral perturbation theory) has emerged. Its key element is a separation of longand short-distance dynamics and an ordering scheme in powers of small momenta. At nuclear matter saturation density $\rho_{0} \simeq 0.16 \mathrm{fm}^{-3}$ the Fermi momentum $k_{f 0}$ and the pion mass $m_{\pi}$ are comparable scales $\left(k_{f 0} \simeq 2 m_{\pi}\right)$, and therefore pions must be included as explicit degrees of freedom in the description of the nuclear many-body dynamics. The contributions to the energy per particle $\bar{E}\left(k_{f}\right)$ of isospin-symmetric nuclear matter as they originate from chiral pion-nucleon dynamics have been computed up to three-loop order in refs. 11, 2. Both calculations are able

\footnotetext{
${ }^{1}$ Work supported in part by BMBF and GSI.
} 
to reproduce correctly the empirical saturation point of nuclear matter by adjusting one single parameter (either a coupling $g_{0}+g_{1} \simeq 3.23$ [1] or a cut-off $\Lambda \simeq 0.65 \mathrm{GeV}$ [2]) related to unresolved short-distance dynamics. The novel mechanism for saturation in these approaches is a repulsive contribution to the energy per particle generated by Pauli-blocking in second order (iterated) one-pion exchange. As outlined in section 2.5 of ref. 2] this mechanism becomes particularly transparent by taking the chiral limit $m_{\pi}=0$. In that case the interaction contributions to the energy per particle are completely summarized by an attractive $k_{f}^{3}$-term and a repulsive $k_{f}^{4}$-term where the parameter-free prediction for the coefficient of the latter is very close to the one extracted from a realistic nuclear matter equation of state.

The single-particle properties, represented by a complex-valued momentum and density dependent nucleon selfenergy $U\left(p, k_{f}\right)+i W\left(p, k_{f}\right)$, have been computed within our approach [2] in ref. 3]. The resulting potential depth $U\left(0, k_{f 0}\right)=-53.2 \mathrm{MeV}$ is in good agreement with that of the empirical nuclear shell [4] or optical model [5]. However, the momentum dependence of the real single-particle potential $U\left(p, k_{f 0}\right)$ with its up- and downward bending (see Fig. 3 in ref. [3] ) turns out to be too strong. As a consequence, the nominal value of the effective nucleon mass at the Fermi surface $p=k_{f 0}$ would be much too high: $M^{*}\left(k_{f 0}\right) \simeq 3 M$. On the other hand, the single-particle properties around the Fermi surface are decisive for the spectrum of thermal excitations and therefore they crucially influence the low temperature behavior of isospin-symmetric nuclear matter. The rather high critical temperature $T_{c} \simeq 25.5 \mathrm{MeV}$ for the liquid-gas phase transition obtained in ref. [6] is a visible manifestation of this intimate relationship.

While there is obviously room and need for improvement in our approach, one must also note at the same time that the single-particle properties in the scheme of Lutz et al.[1] (where explicit short-range terms are iterated with pion-exchange) come out completely unrealistic [7]. The potential depth of $U\left(0, k_{f 0}\right)=-20 \mathrm{MeV}$ is by far too weakly attractive. Most seriously, the total single-particle energy $T_{\text {kin }}(p)+U\left(p, k_{f 0}\right)$ does not rise monotonically with the nucleon momentum $p$, thus implying a negative effective nucleon mass at the Fermi surface (see Fig. 3 in ref. [7]). This ruins the behavior of nuclear matter at finite temperatures since a critical temperature of $T_{c}>40 \mathrm{MeV}$ exceeds acceptable values by more than a factor of two.

The isospin properties of nuclear matter have also been investigated in ref. [2]. The prediction for the asymmetry energy at saturation density $A\left(k_{f 0}\right)=33.8 \mathrm{MeV}$ is in good agreement with its empirical value. However, one finds a downward bending of $A\left(k_{f}\right)$ at densities $\rho>0.2 \mathrm{fm}^{-3}$. Such a behavior of the asymmetry energy $A\left(k_{f}\right)$ is presumably not realistic. The energy per particle of pure neutron matter $\bar{E}_{n}\left(k_{n}\right)$ as a function of the neutron density $\rho_{n}=k_{n}^{3} / 3 \pi^{2}$ shows a similar downward bending behavior (see Fig. 8 in ref. [2]) and at lower neutron densities, there is only rough agreement with realistic neutron matter calculations. The mere fact that neutron matter came out to be unbound in ref. 2] with no further adjusted parameter was however nontrivial. The isospin properties of nuclear matter in the scheme of Lutz et al. [1] with a second free parameter adjusted, are qualitatively the same. The dashed curves in Figs. 6,7 of ref. [7] display a downward bending of $\bar{E}_{n}\left(k_{n}\right)$ and $A\left(k_{f}\right)$ at even lower densities, $\rho>0.15 \mathrm{fm}^{-3}$. An extended version of that approach with pion-exchange plus two zero-range NN-contact interactions iterated to second order and in total four adjustable parameters has been studied recently in ref. [8]. The finding of that work is that within such a complete fourth order calculation (thus exhausting all possible terms up-to-and-including $\mathcal{O}\left(k_{f}^{4}\right)$ ) there is no optimal set of the four short-range parameters with which one could reproduce simultaneously and accurately all semiempirical properties of nuclear matter. The conditions for a good neutron matter equation of state and equally good single-particle properties (and consequently a realistic finite temperature behavior) are in fact mutually exclusive in that approach.

Calculations of nuclear matter based on the universal low-momentum nucleon-nucleon potential $V_{\text {low-k }}$ have recently been performed in ref. [9]. The results obtained so far in Hartree-Fock 
or Brueckner-Hartree-Fock approximation are unsatisfactory (see Figs. 1,2 in ref. [9]), since no saturation occurs in the equation of state. It has been concluded that for the potential $V_{\text {low }-\mathrm{k}}$ the Brueckner-Hartree-Fock approximation is applicable only at very low densities. These findings together with the identification of a successful saturation mechanism in the chiral approaches hint at the fact that the Brueckner ladder does not generate all relevant medium modifications which set in already at very low densities (typically at about one-tenth the equilibrium density of nuclear matter, corresponding to Fermi momenta around $k_{f} \simeq m_{\pi}$ ).

Up to this point the situation can be summarized as follows. Chiral two-pion exchange restricted to nucleon intermediate states (basically the second-order spin-spin and tensor force plus Pauli blocking effects), together with a single (finetuned) contact-term representing shortdistance dynamics, is already surprisingly successful in binding and saturating nuclear matter and reproducing key properties such as the asymmetry energy and the compression modulus. However, the detailed behavior of the nucleon single-particle potential and the density of states at the Fermi surface are not well described at this order in the small-momentum expansion.

Let us also comment on the relationship between our appoach to nuclear matter and the effective field theory treatment of (free) NN-scattering [10, 11, 12. We are including to the respective order the same long-range components from (one- and) two-pion exchange. The shortrange NN-contact terms are however treated differently. Instead of introducing these terms in a potential which is then iterated in a Lippmann-Schwinger equation, we adjust their strengths (in a perturbative calculation) to a few empirical nuclear matter properties. The resulting values of the coupling constants are therefore not directly comparable with those of the NN-potential. We note also that the important role of chiral two-pion exchange for peripheral NN-scattering is well established [11, 12, 13.

From the point of view of the driving pion-nucleon dynamics the previously mentioned chiral calculations of nuclear matter 1, 2, 8, are indeed still incomplete. They include only (S- and) Pwave Born terms but leave out the excitation of the spin-isospin-3/2 $\Delta$ (1232)-resonance, which is the prominent feature of low-energy $\pi N$-scattering. It is also well known that the two-pion exchange between nucleons with excitation of virtual $\Delta$-isobars generates most of the needed isoscalar central NN-attraction. In phenomenological one-boson exchange models this part of the $\mathrm{NN}$-interaction is often simulated by a fictitious " $\sigma$ "-meson exchange. A parameter-free calculation of the isoscalar central potential $\widetilde{V}_{C}(r)$ generated by $2 \pi$-exchange with single and double $\Delta$-excitation in ref. [14] (see Fig. 2 therein) agrees almost perfectly with the phenomenological " $\sigma$ "-exchange potential at distances $r>2 \mathrm{fm}$, but not at shorter distances. The more detailed behavior of the $2 \pi$-exchange isoscalar central potential with single virtual $\Delta$-excitation is reminiscent of the van-der-Waals potential. It has the form [14]:

$$
\widetilde{V}_{C}^{(N \Delta)}(r)=-\frac{3 g_{A}^{4}}{64 \pi^{2} f_{\pi}^{4} \Delta} \frac{e^{-2 x}}{r^{6}}\left(6+12 x+10 x^{2}+4 x^{3}+x^{4}\right),
$$

with $x=m_{\pi} r$ and the prefactor includes the spin-isospin (axial) polarizability of the nucleon [15], $g_{A}^{2} / f_{\pi}^{2} \Delta=5.2 \mathrm{fm}^{3}$, from the virtual $N \rightarrow \Delta(1232) \rightarrow N$ transition. The familiar $r^{-6}$ dependence of the non-relativistic van-der-Waals interaction emerges in the chiral limit, $m_{\pi}=0$.

A consideration of mass scales also suggests to include the $\Delta(1232)$-isobar as an explicit degree of freedom in nuclear matter calculations. The delta-nucleon mass splitting of $\Delta=293 \mathrm{MeV}$ is comparable to the Fermi momentum $k_{f 0} \simeq 262 \mathrm{MeV}$ at nuclear matter saturation density. Propagation effects of virtual $\Delta(1232)$-isobars can therefore be resolved at the densities of interest. Based on these scale arguments we adopt a calculational scheme in which we count the Fermi momentum $k_{f}$, the pion mass $m_{\pi}$ and the $\Delta N$-mass splitting $\Delta$ simultaneously as "small scales". The non-relativistic treatment of the nuclear matter many-body problem naturally goes 
conform with such an expansion in powers of small momenta. Relativistic corrections are relegated to higher orders in this expansion scheme. The leading contributions from $2 \pi$-exchange with virtual $\Delta$-excitation to the energy per particle (or the single-particle potential) are generically of fifth power in the small momenta $\left(k_{f}, m_{\pi}, \Delta\right)$. With respect to the counting in small momenta the effects from irreducible $2 \pi$-exchange evaluated in ref. 22, 3, 6] belong to the same order. However, since the $\pi N \Delta$-coupling constant is about twice as large as the $\pi N N$-coupling constant one can expect that the $\Delta$-driven $2 \pi$-exchange effects are the dominant ones. The importance of $\Delta(1232)$-degrees of freedom has also been pointed out in the "ab-initio" calculations of the Illinois group [16, 17.

The purpose of the present paper is to present a calculation of (isospin-symmetric and isospin-asymmetric) nuclear matter which includes systematically all effects from $2 \pi$-exchange with virtual $\Delta$-excitation up to three-loop order in the energy density. The contributions to the energy per particle (or the single-particle potential) can be classified as two-body terms and three-body terms. Two-body terms can be directly expressed through the NN-scattering T-matrix (i.e. the NN-potential in momentum space). Three-body terms on the other hand can be interpreted as Pauli-blocking effects on the two-body terms imposed by the filled Fermi-sea of nucleons. The notion of "three-body term" is taken here in a more general context, namely in the sense that three nucleons in the Fermi sea participate in interactions. The NN T-matrix generated by the in general ultra-violet divergent pion-loop diagrams requires regularization (and renormalization). We adopt here a suitably subtracted dispersion-relation representation of the T-matrix where this procedure is accounted for by a few subtraction constants. The latter constants are understood to encode unresolved short-distance NN-dynamics. The associated $k_{f^{-}}^{3}$ and $k_{f}^{5}$-terms in the energy per particle are then adjusted to some empirical property of nuclear matter (e.g. the maximal binding energy of $16 \mathrm{MeV}$ ).

Our paper is organized as follows: In section 2 we start with the equation of state of isospinsymmetric nuclear matter. We evaluate analytically the three-loop in-medium diagrams generated by the chiral $\pi N \Delta$-dynamics and perform the necessary adjustment of short-range parameters. Section 3 deals with the real single-particle potential $U\left(p, k_{f}\right)$ whose improved momentum dependence turns out to be a true prediction. In section 4 we reconsider the imaginary singleparticle potential $W\left(p, k_{f}\right)$ of ref. [3] , but now extended into the region above the Fermi surface

$p>k_{f}$. Section 5 is devoted to the effective nucleon mass $\widetilde{M}^{*}(\rho)$ and the strength function of the $(\vec{\nabla} \rho)^{2}$-term in the nuclear energy density functional. In section 6 we extend our calculation of isospin-symmetric nuclear matter to finite temperatures $T$. The main interest lies there on the critical temperature $T_{c}$ of the first-order liquid-gas phase transition for which we find an improved value of $T_{c} \simeq 15 \mathrm{MeV}$. Sections 7, 8 and 9 deal with the equation of state of pure neutron matter $\bar{E}_{n}\left(k_{n}\right)$, the asymmetry energy $A\left(k_{f}\right)$ and the isovector single-particle potential $U_{I}\left(p, k_{f}\right)$. These three quantities reveal the isospin properties of the underlying $\pi N \Delta$-dynamics. Explicit inclusion of the $\Delta(1232)$-degree of freedom leads to a substantial improvement: the notorious downward bending of $\bar{E}_{n}\left(k_{n}\right)$ and $A\left(k_{f}\right)$ observed in previous chiral calculations is now eliminated. Finally, section 10 ends with a summary and some concluding remarks.

\section{Equation of state of isospin-symmetric nuclear matter}

We start the discussion with the equation of state of isospin-symmetric nuclear matter for which one has a fairly good knowledge of the empirical saturation point. We first write down the contributions to the energy per particle $\bar{E}\left(k_{f}\right)$ as they arise from $2 \pi$-exchange with single and double virtual $\Delta$-isobar excitation.

Fig. 1 shows the relevant one-loop triangle, box and crossed box diagrams contributing to 

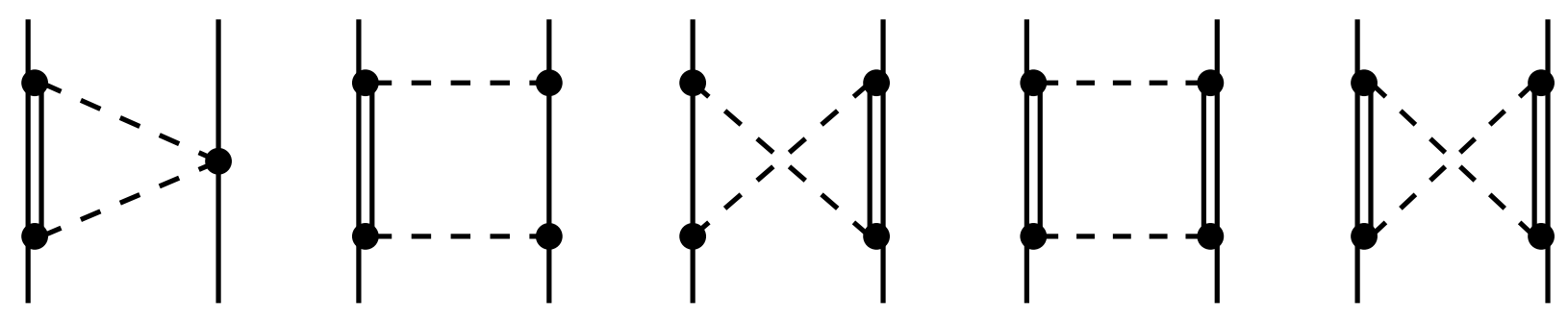

Figure 1: One-loop two-pion exchange diagrams with single and double $\Delta(1232)$-isobar excitation. Diagrams for which the role of both nucleons is interchanged are not shown.

the NN T-matrix (or the momentum space potential). The finite parts of these diagrams have been evaluated analytically in section 3 of ref. 14 employing the usual non-relativistic $\Delta \leftrightarrow \pi N$ transition vertices and $\Delta$-propagator (see eq.(4) in ref. 14]). By closing the two open nucleon lines to either two rings or one ring one gets (in diagrammatic representation) the Hartree or Fock contribution to the energy density of nuclear matter. The Hartree contribution to the energy per particle evidently goes linear with the nucleon density $\rho=2 k_{f}^{3} / 3 \pi^{2}$, namely $\bar{E}\left(k_{f}\right)^{(2 H)}=$ $-V_{C}(0) \rho / 2$ with $V_{C}(0)$ the isoscalar central NN-amplitude at zero momentum transfer [14]. The Fock contribution on the other hand is obtained by integrating the spin- and isospin-contracted T-matrix (depending on the momentum transfer variable $\left|\vec{p}_{1}-\vec{p}_{2}\right|$ ) over the product of two Fermi spheres $\left|\vec{p}_{1,2}\right|<k_{f}$ of radius $k_{f}$. We separate regularization dependent short-range parts in the T-matrix (originating from the divergences of the loop diagrams) from the unique longrange terms with the help of a twice-subtracted dispersion relation. The resulting subtraction constants give rise to a contribution to the energy per particle of the form:

$$
\bar{E}\left(k_{f}\right)^{(c t)}=B_{3} \frac{k_{f}^{3}}{M^{2}}+B_{5} \frac{k_{f}^{5}}{M^{4}},
$$

where $B_{3}$ and $B_{5}$ are chosen for convenience as dimensionless. $M=939 \mathrm{MeV}$ stands for the (average) nucleon mass. Note that eq.(1) is completely equivalent to the contribution of a momentum independent and $p^{2}$-dependent $\mathrm{NN}$-contact interaction. We interpret the parameters $B_{3,5}$ to subsume all unresolved short-distance NN-dynamics relevant for isospin-symmetric nuclear matter at low and moderate densities. The long-range parts of the $2 \pi$-exchange (two-body) Fock diagrams can be expressed as:

$$
\begin{aligned}
\bar{E}\left(k_{f}\right)^{(2 F)}= & \frac{1}{8 \pi^{3}} \int_{2 m_{\pi}}^{\infty} d \mu \operatorname{Im}\left(V_{C}+3 W_{C}+2 \mu^{2} V_{T}+6 \mu^{2} W_{T}\right)\left\{3 \mu k_{f}-\frac{4 k_{f}^{3}}{3 \mu}\right. \\
& \left.+\frac{8 k_{f}^{5}}{5 \mu^{3}}-\frac{\mu^{3}}{2 k_{f}}-4 \mu^{2} \arctan \frac{2 k_{f}}{\mu}+\frac{\mu^{3}}{8 k_{f}^{3}}\left(12 k_{f}^{2}+\mu^{2}\right) \ln \left(1+\frac{4 k_{f}^{2}}{\mu^{2}}\right)\right\}
\end{aligned}
$$

where $\operatorname{Im} V_{C}, \operatorname{Im} W_{C}, \operatorname{Im} V_{T}$ and $\operatorname{Im} W_{T}$ are the spectral functions of the isoscalar and isovector central and tensor NN-amplitudes, respectively. Explicit expressions of these imaginary parts for the contributions of the triangle diagram with single $\Delta$-excitation and the box diagrams with single and double $\Delta$-excitation can be easily constructed from the analytical formulas given in section 3 of ref. [14]. The $\mu$ - and $k_{f}$-dependent weighting function in eq.(2) takes care that at low and moderate densities this spectral integral is dominated by low invariant $\pi \pi$-masses $2 m_{\pi}<\mu<1 \mathrm{GeV}$. The contributions to the energy per particle from irreducible $2 \pi$-exchange (with only nucleon intermediate states) can also be cast into the form eq.(2). The corresponding 
the (right) three-body Fock diagram in Fig. 2 to the energy per particle reads:

$$
\bar{E}\left(k_{f}\right)^{(3 F)}=-\frac{3 g_{A}^{4} m_{\pi}^{6} u^{-3}}{4 \Delta\left(4 \pi f_{\pi}\right)^{4}} \int_{0}^{u} d x\left[2 G_{S}^{2}(x, u)+G_{T}^{2}(x, u)\right],
$$

where we have introduced the two auxiliary functions:

$$
\begin{aligned}
G_{S}(x, u)= & \frac{4 u x}{3}\left(2 u^{2}-3\right)+4 x[\arctan (u+x)+\arctan (u-x)] \\
& +\left(x^{2}-u^{2}-1\right) \ln \frac{1+(u+x)^{2}}{1+(u-x)^{2}}, \\
G_{T}(x, u)= & \frac{u x}{6}\left(8 u^{2}+3 x^{2}\right)-\frac{u}{2 x}\left(1+u^{2}\right)^{2} \\
+ & \frac{1}{8}\left[\frac{\left(1+u^{2}\right)^{3}}{x^{2}}-x^{4}+\left(1-3 u^{2}\right)\left(1+u^{2}-x^{2}\right)\right] \ln \frac{1+(u+x)^{2}}{1+(u-x)^{2}} .
\end{aligned}
$$

Evidently, the three-body Fock term in eq.(6) is attractive. The remaining contributions to $\bar{E}\left(k_{f}\right)$ from the (relativistically improved) kinetic energy, from the $1 \pi$-exchange Fock diagram and from the iterated $1 \pi$-exchange Hartree and Fock diagrams have been written down in eqs.(5-11) of ref. 2]. The strongly attractive contribution from iterated $1 \pi$-exchange linear in the density and the cutoff $\Lambda$ (see eq.(15) in ref.[2]) is now of course not counted extra since $B_{3}$ in eq.(1) collects all such possible terms. Adding all pieces we arrive at the full energy per particle $\bar{E}\left(k_{f}\right)$ at three-loop order. It involves three parameters, $B_{3}$ and $B_{5}$ of the two-body contact term eq.(1) and $\zeta$ which controls a three-body contact term in eq. (5).

Let us first look at generic properties of the nuclear matter equation of state in our calculation. Binding and saturation occurs in a wide range of the two adjustable parameters $B_{3,5}$. However, with the full strength $(\zeta=0)$ of the repulsive $\rho^{2}$-term from the $2 \pi$-exchange threebody Hartree diagram (see eq.(5)) the saturation curve rises much too steeply with increasing density. This causes a too low saturation density $\rho_{0}$ and a too high nuclear matter compressibility, $K>350 \mathrm{MeV}$. We cure this problem in a minimal way by introducing an attractive three-body contact term. With $\zeta=-3 / 4$ the remaining repulsive $\rho^{2}$-term in eq.(5) gets canceled by an analogous attractive contribution from the three-body Fock diagram. Clearly, the need for introducing an attractive three-body contact term into our calculation points to some short-distance physics whoose dynamical origin lies outside the present framework of perturbative chiral pion-nucleon interactions. It will become clear in the following sections that the predictive power of our calculation is nevertheless not reduced by this procedure.

We fix the minimum of the saturation curve $\bar{E}\left(k_{f}\right)$ to the value $\bar{E}_{0}=-16.0 \mathrm{MeV}$. With $B_{3}$ adjusted to the value $B_{3}=-7.99$ and $B_{5}$ taken to be zero, $B_{5}=0$, the full line in Fig. 3 results. The predicted value of the saturation density is $\rho_{0}=0.157 \mathrm{fm}^{-3}$, corresponding to a Fermi momentum of $k_{f 0}=261.6 \mathrm{MeV}=1.326 \mathrm{fm}^{-1}$. This is very close to the semi-empirical value $\rho_{0}=0.158 \mathrm{fm}^{-3}$ obtained by extrapolation from inelastic electron scattering off heavy nuclei [19]. The decomposition of the negative binding energy $\bar{E}_{0}=-16.0 \mathrm{MeV}$ into contributions of second, third, fourth and fifth power in small momenta reads: $\bar{E}_{0}=(21.9-145.5+107.8-0.2) \mathrm{MeV}$ with the typical balance between large third and fourth order terms [2]. The very small fifth order term splits furthermore as $(-13.8+13.6) \mathrm{MeV}$ into the contribution from the three-body contactinteraction (proportional to $\zeta=-3 / 4$ ) and a remainder. Evidently, since $\bar{E}_{0}=-16.0 \mathrm{MeV}$ is a small number that needs to be finetuned in our calculation there remains the question of the "convergence" of the small momentum expansion. The nuclear matter compressibility $K=k_{f 0}^{2} \bar{E}^{\prime \prime}\left(k_{f 0}\right)$ related to the curvature of the saturation curve at its minimum comes out 


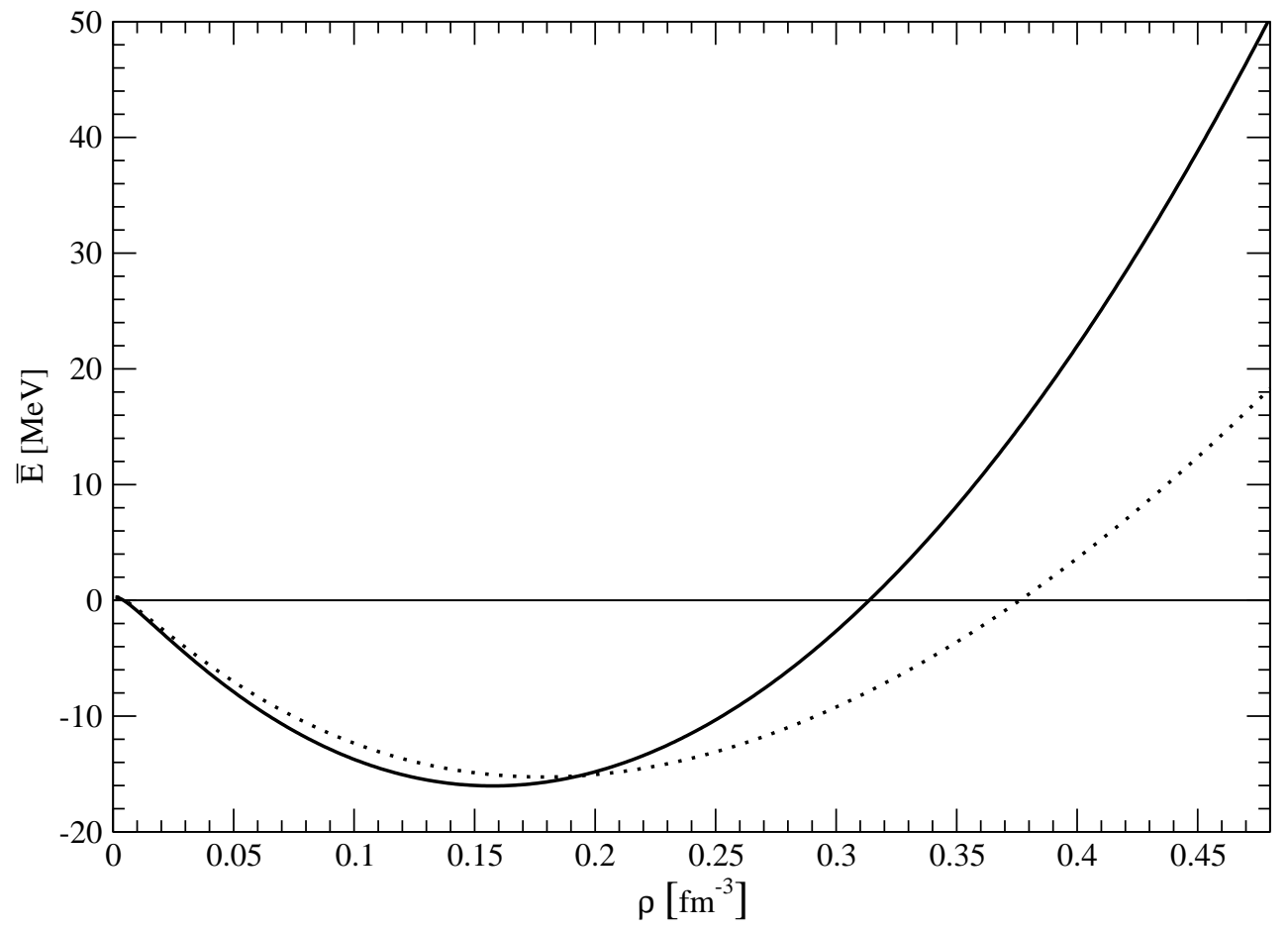

Figure 3: The energy per particle $\bar{E}\left(k_{f}\right)$ of isospin-symmetric nuclear matter as a function of the nucleon density $\rho=2 k_{f}^{3} / 3 \pi^{2}$. The dashed line refers to the result of ref.[2], with only pions and nucleons as active degrees of freedom. The full line includes effects from $2 \pi$-exchange with virtual $\Delta$-excitation. The short-range parameters are $B_{3}=-7.99$ and $B_{5}=0$.

as $K=304 \mathrm{MeV}$. This number is somewhat high but still acceptable, since it exceeds e.g. the value $K=272 \mathrm{MeV}$ obtained in the relativistic mean-field model of ref. [20] only by $12 \%$. Inspection of Fig. 3 shows that the saturation curve is well approximated by a shifted parabola $\bar{E}\left(k_{f}\right)=\bar{E}_{0}\left(2 \rho_{0}-\rho\right) \rho / \rho_{0}^{2}$ with a second zero-crossing at twice nuclear matter density $2 \rho_{0}$. This leads to a compressibility estimate of $K=-18 \bar{E}_{0}=288 \mathrm{MeV}$, not far from the calculated value. We have also studied variations of the parameter $B_{5}$. Within the limits set by a stable saturation point (and realistic single-particle properties, see next section), the effects on the compressibility $K$ are marginal (less than $10 \mathrm{MeV}$ reduction). Therefore we stay with the minimal choice $B_{5}=0$ (together with $B_{3}=-7.99$ and $\zeta=-3 / 4$ ). The dashed line in Fig. 3 shows for comparison the equation of state resulting from our previous chiral calculation 2 with no $\pi N \Delta$-dynamics included. In that work the saturation density $\rho_{0}=0.178 \mathrm{fm}^{-3}$ came out somewhat too high, but the compressibility $K=255 \mathrm{MeV}$ had a better value. The stronger rise of the full curve in Fig. 3 with density $\rho$ is a consequence of including higher order terms in the (small-momentum) $k_{f}$-expansion.

\section{Real part of single-particle potential}

In this section we discuss the real part $U\left(p, k_{f}\right)$ of the single-particle potential. As outlined in ref. [3] the contributions to the (real) nuclear mean-field $U\left(p, k_{f}\right)$ can be classified as two-body and three-body potentials. The parameters $B_{3,5}$ introduced in eq.(1) reappear in a contribution to the two-body potential of the form:

$$
U_{2}\left(p, k_{f}\right)^{(c t)}=2 B_{3} \frac{k_{f}^{3}}{M^{2}}+B_{5} \frac{k_{f}^{3}}{3 M^{4}}\left(3 k_{f}^{2}+5 p^{2}\right) .
$$


Its density- and momentum-dependence is completely fixed by the Hugenholtz-Van-Hove theorem [21] and a sum rule which connects it to the energy per particle $\bar{E}\left(k_{f}\right)^{(c t)}$ (see eqs. $(5,7)$ in ref. [3]). The Fock diagrams of $2 \pi$-exchange with virtual $\Delta$-excitation give rise to a contribution to the two-body potential which can be written as a (subtracted) dispersion integral:

$$
\begin{aligned}
U_{2}\left(p, k_{f}\right)^{(F)}= & \frac{1}{2 \pi^{3}} \int_{2 m_{\pi}}^{\infty} d \mu \operatorname{Im}\left(V_{C}+3 W_{C}+2 \mu^{2} V_{T}+6 \mu^{2} W_{T}\right) \\
& \times\left\{\mu k_{f}+\frac{2 k_{f}^{3}}{15 \mu^{3}}\left(3 k_{f}^{2}+5 p^{2}\right)-\frac{2 k_{f}^{3}}{3 \mu}-\mu^{2} \arctan \frac{k_{f}+p}{\mu}\right. \\
& \left.-\mu^{2} \arctan \frac{k_{f}-p}{\mu}+\frac{\mu}{4 p}\left(\mu^{2}+k_{f}^{2}-p^{2}\right) \ln \frac{\mu^{2}+\left(k_{f}+p\right)^{2}}{\mu^{2}+\left(k_{f}-p\right)^{2}}\right\} .
\end{aligned}
$$

By opening a nucleon line in the three-body diagrams of Fig. 2 one gets (per diagram) three different contributions to the three-body potential. In the case of the (left) Hartree diagram they read altogether:

$$
\begin{aligned}
U_{3}\left(p, k_{f}\right)^{(H)}= & \frac{g_{A}^{4} m_{\pi}^{6}}{\Delta\left(2 \pi f_{\pi}\right)^{4}}\left\{2 u^{6}(1+\zeta)+u^{2}-7 u^{4}-\frac{1}{4}\left(1+9 u^{2}\right) \ln \left(1+4 u^{2}\right)\right. \\
& +5 u^{3}[\arctan 2 u+\arctan (u+x)+\arctan (u-x)] \\
& \left.+\frac{u^{3}}{2 x}\left(2 x^{2}-2 u^{2}-3\right) \ln \frac{1+(u+x)^{2}}{1+(u-x)^{2}}\right\}
\end{aligned}
$$

with the abbreviation $x=p / m_{\pi}$. Note that the paramter $\zeta=-3 / 4$ related to the threebody contact-interaction has no influence on the momentum dependence of the single-particle potential. On the other hand the (right) Fock diagram in Fig. 2 generates a total contribution to the three-body potential of the form:

$$
\begin{aligned}
U_{3}\left(p, k_{f}\right)^{(F)}= & -\frac{g_{A}^{4} m_{\pi}^{6} x^{-2}}{4 \Delta\left(4 \pi f_{\pi}\right)^{4}}\left\{2 G_{S}^{2}(x, u)+G_{T}^{2}(x, u)\right. \\
& \left.+\int_{0}^{u} d \xi\left[4 G_{S}(\xi, u) \frac{\partial G_{S}(\xi, x)}{\partial x}+2 G_{T}(\xi, u) \frac{\partial G_{T}(\xi, x)}{\partial x}\right]\right\},
\end{aligned}
$$

with $G_{S, T}(x, u)$ defined in eqs. $(7,8)$. The real single-particle potential $U\left(p, k_{f}\right)$ is completed by adding to the terms eqs. (9-12) the contributions from $1 \pi$-exchange and iterated $1 \pi$-exchange written down in eqs. (8-13) of ref. [3]. The slope of the real single-particle potential $U\left(p, k_{f}\right)$ at the Fermi surface $p=k_{f}$ determines the effective nucleon mass (in the nomenclature of ref.[22], the product of " $k$-mass" and " $E$-mass" divided by the free nucleon mass $M=939 \mathrm{MeV}$ ) via a relation:

$$
M^{*}\left(k_{f}\right)=M\left[1-\frac{k_{f}^{2}}{2 M^{2}}+\left.\frac{M}{k_{f}} \frac{\partial U\left(p, k_{f}\right)}{\partial p}\right|_{p=k_{f}}\right]^{-1} .
$$

The second term $-k_{f}^{2} / 2 M^{2}$ in the square brackets stems from the relativistic correction $-p^{4} / 8 M^{3}$ to the kinetic energy.

The full line in Fig. 4 shows the real part of the single-particle potential $U\left(p, k_{f 0}\right)$ at saturation density $k_{f 0}=261.6 \mathrm{MeV}$ as a function of the nucleon momentum $p$. The dotted line includes in addition the relativistically improved kinetic energy $T_{\text {kin }}(p)=p^{2} / 2 M-p^{4} / 8 M^{3}$. With the parameters $B_{5}=0, \zeta=-3 / 4$ fixed and $B_{3}=-7.99$ adjusted in section 1 to the binding energy at equilibrium we find a potential depth of $U\left(0, k_{f 0}\right)=-78.2 \mathrm{MeV}$. This is very close to the result $U\left(0, k_{f 0}\right) \simeq-80 \mathrm{MeV}$ of the relativistic Dirac-Brueckner approach of ref. [23]. 


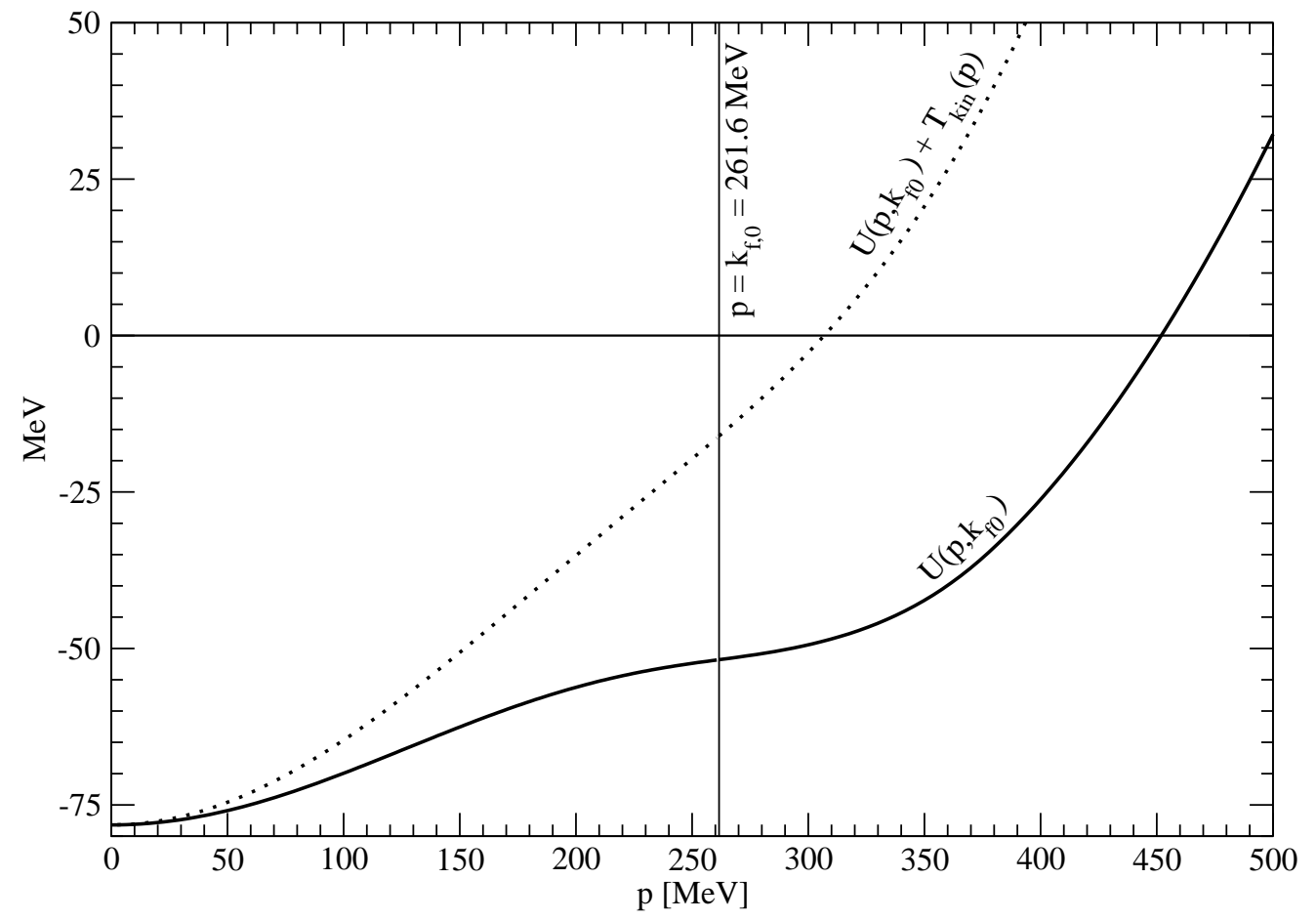

Figure 4: Full line: real part of the single-particle potential $U\left(p, k_{f 0}\right)$ at saturation density $k_{f 0}=261.6 \mathrm{MeV}$ as a function of the nucleon momentum $p$. The dotted line includes in addition the relativistically improved kinetic energy $T_{\text {kin }}(p)=p^{2} / 2 M-p^{4} / 8 M^{3}$. Both curves are extended into the region above the Fermi surface $p>k_{f 0}$.

For comparison, the calculation of ref. 24] based on the phenomenological Paris NN-potential finds a somewhat shallower potential depth of $U\left(0, k_{f 0}\right) \simeq-64 \mathrm{MeV}$. One observes that with the chiral $\pi N \Delta$-dynamics included, the real single-particle potential $U\left(p, k_{f 0}\right)$ grows monotonically with the nucleon momentum $p$. The downward bending above $p=180 \mathrm{MeV}$ displayed in Fig. 3 of ref. [3] is now eliminated. The slope at the Fermi surface $p=k_{f 0}$ translates into an effective nucleon mass of $M^{*}\left(k_{f 0}\right)=0.88 M$. This is now a realistic value compared to $M^{*}\left(k_{f 0}\right) \simeq 3 M$ obtained in our previous calculation [3, 6] without any explicit $\Delta$-isobars. Note also that the chiral approach of ref. [8] (where both explicit short-range terms and pion-exchange are iterated) has found the lower bound $M^{*}\left(k_{f 0}\right)>1.4 M$.

The dotted curve in Fig. 4 for the total single-particle energy $T_{\text {kin }}(p)+U\left(p, k_{f 0}\right)$ hits the value $\bar{E}\left(k_{f 0}\right)=\bar{E}_{0}=-16 \mathrm{MeV}$ at the Fermi surface $p=k_{f 0}$, as required by the Hugenholtz-Van-Hove theorem [21]. This important theorem holds strictly in our (perturbative) calculation, whereas (non-perturbative) Brueckner-Hartree-Fock approaches often fail to respect it [25]. In Fig. 4 we have also extended both curves into the region above the Fermi surface $p>k_{f 0}$. In general this extension is not just an analytical continuation of the potential from below the Fermi surface. Whereas eqs.(9-12) for the contributions from $2 \pi$-exchange with $\Delta$-excitation apply in both regions, there are non-trivial changes in the expressions from iterated $1 \pi$-exchange. These modifications are summarized in the Appendix. The smooth rise of $U\left(p, k_{f 0}\right)$ as it crosses the Fermi surface and proceeds up to $p \simeq 400 \mathrm{MeV}$ is compatible with other calculations [23, 24]. Beyond this momentum scale one presumably exceeds the limits of validity of the present chiral perturbation theory calculation of nuclear matter.

The full line in Fig. 5 shows the potential depth $U\left(0, k_{f}\right)$ for a nucleon at the bottom of the Fermi sea as a function of the nucleon density $\rho=2 k_{f}^{3} / 3 \pi^{2}$. The band spanned by the 


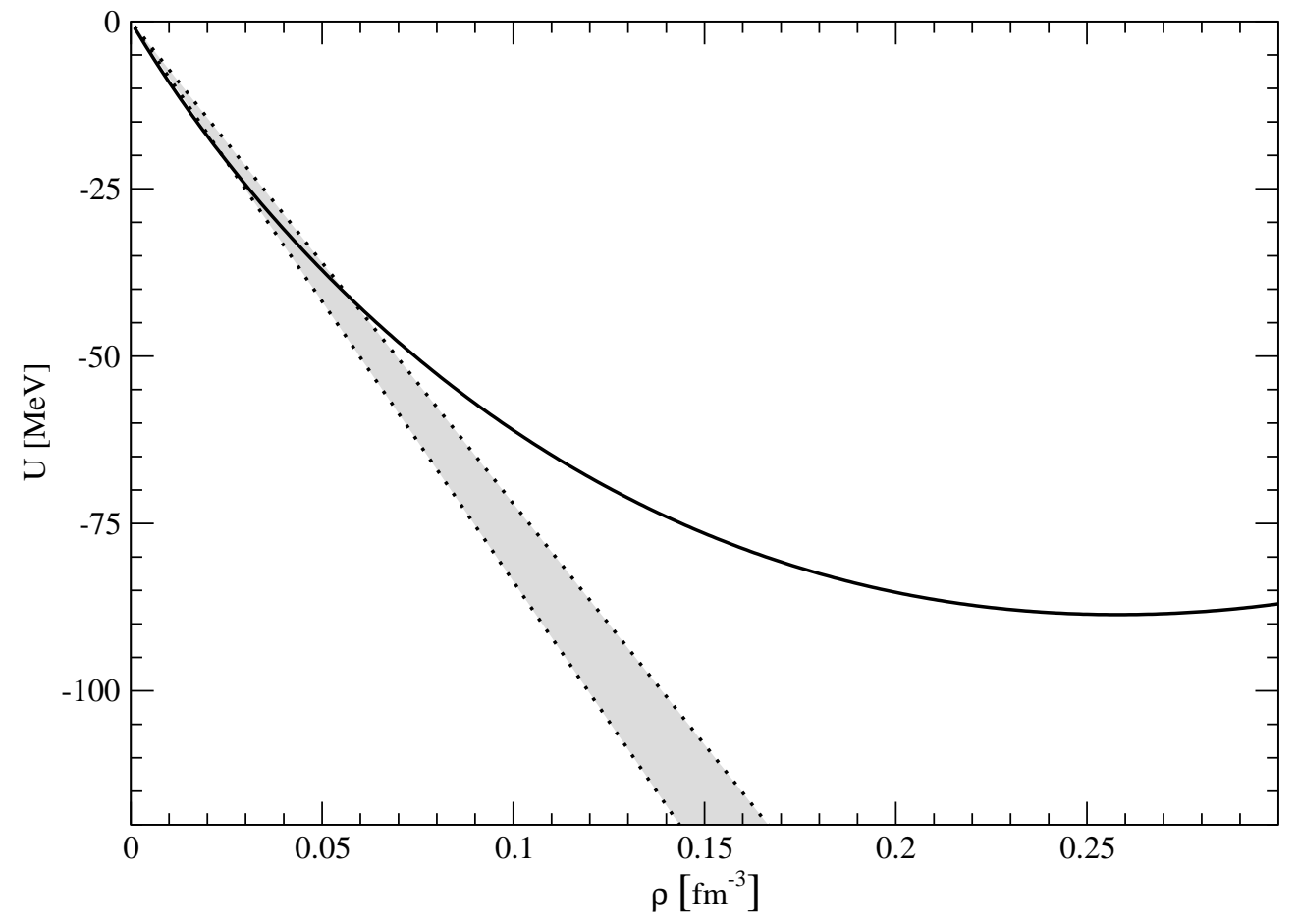

Figure 5: The full line shows the real part of the single-particle potential $U\left(0, k_{f}\right)$ at nucleon momentum $p=0$ versus the density $\rho=2 k_{f}^{3} / 3 \pi^{2}$. The band is obtained from the universal low-momentum NN-potential $V_{\text {low-k }}$ in linear density approximation.

dotted lines stems from the universal low-momentum NN-potential $V_{\text {low-k }}$ [26] in linear density approximation. In this approximation the potential depth simply reads:

$$
U\left(0, k_{f}\right)=\frac{3 \pi \rho}{2 M}\left[V_{\mathrm{low}-\mathrm{k}}^{\left({ }^{1} S_{0}\right)}(0,0)+V_{\mathrm{low}-\mathrm{k}}^{\left({ }^{3} S_{1}\right)}(0,0)\right]
$$

with $V_{\text {low-k }}^{\left({ }^{1} S_{0}\right)}(0,0) \simeq-1.9 \mathrm{fm}$ and $V_{\text {low-k }}^{\left({ }^{3} S_{1}\right)}(0,0) \simeq-(2.2 \pm 0.3) \mathrm{fm}$ [26, 27, 28, the two S-wave potentials at zero momentum. It is interesting to observe that both potential depths agree fairly well at low densities, $\rho \leq 0.07 \mathrm{fm}^{-3}$. This agreement is by no means trivial since $V_{\text {low }-\mathrm{k}}$ is constructed to reproduce accurately the low-energy NN-scattering data (phase-shifts and mixing angles) whereas our adjustment of the $B_{3}$-term (linear in density) is made at saturation density $\rho_{0}=0.157 \mathrm{fm}^{-3}$. It is evident from Fig. 5 that a linear extrapolation does not work from zero density up to nuclear matter saturation density. Strong curvature effects set in already at Fermi momenta around $k_{f} \simeq m_{\pi}$ if (and only if) pion-dynamics is treated explicitly. ${ }^{2}$ We note also that an "improved" determination of the potential depth $U\left(0, k_{f}\right)$ from $V_{\text {low-k }}$ where one takes into account its momentum dependence in the repulsive Fock contribution leads to concave curves which bend below the straight dotted lines in Fig. 5. In the case $V_{\text {low-k }}^{\left({ }^{3} S_{1}\right)}(0,0)=-1.9 \mathrm{fm}$ the potential depth $U\left(0, k_{f 0}\right)$ at saturation density would increase to $-132.6 \mathrm{MeV}$ (compared to $-113.4 \mathrm{MeV}$ in linear density approximation). The present observations concerning the potential depth $U\left(0, k_{f}\right)$ may indicate why calculations based on $V_{\text {low-k }}$ did so far not find saturation of nuclear matter 9]. It seems that the Brueckner ladder does not generate all relevant medium modifications which set in already at rather low densities $k_{f} \simeq m_{\pi}$ (if the pion-dynamics is treated explicitly).

\footnotetext{
${ }^{2}$ As example for the extreme inherent non-linearities, consider the formula for the three-body potential $U_{3}\left(0, k_{f}\right)^{(H)}$ in eq.(11). Its mathematical Taylor-series expansion converges only for $k_{f}<m_{\pi} / 2$. This corresponds to tiny densities, $\rho<0.0027 \mathrm{fm}^{-3}$.
} 


\section{Imaginary part of single-particle potential}

In this section, we reconsider the imaginary part $W\left(p, k_{f}\right)$ of the single-particle potential. To the three-loop order we are working here it is still given completely by iterated $1 \pi$-exchange with no contribution from the $\pi N \Delta$-dynamics. The new aspect here is the extension into the region above the Fermi surface $p>k_{f}$, which is not an analytical continuation from below the Fermi surface. As outlined in ref. [3] the contributions to $W\left(p, k_{f}\right)$ can be classified as two-body, three-body and four-body terms. From the Hartree diagram of iterated $1 \pi$-exchange one finds altogether (in the region above the Fermi surface $p>k_{f}$ ):

$$
\begin{aligned}
W\left(p, k_{f}\right)^{(H)}= & \frac{\pi g_{A}^{4} M m_{\pi}^{4}}{\left(4 \pi f_{\pi}\right)^{4}}\left\{\left(9+6 u^{2}+\frac{4 u^{3}}{x}-2 x^{2}\right) \ln \left[1+(u+x)^{2}\right]\right. \\
& +\left(\frac{4 u^{3}}{x}+2 x^{2}-9-6 u^{2}\right) \ln \left[1+(x-u)^{2}\right]+4 u x\left(2-u^{2}\right) \\
& +\frac{1}{x}\left[\left(7+15 u^{2}-15 x^{2}\right)[\arctan (u+x)-\arctan (x-u)]+\frac{12 u^{5}}{5}-\frac{21 u}{2}\right. \\
& \left.-8 u^{3} \ln \left(1+4 u^{2}\right)-\left(15 u^{2}+\frac{7}{4}\right) \arctan 2 u\right]+3 \theta(\sqrt{2} u-x) \int_{y_{\min }}^{u / x} d y\left(x^{2} y^{2}-u^{2}\right) \\
& \times \mathcal{A}_{y}\left[\frac{2 s^{2}+s^{4}}{1+s^{2}}-2 \ln \left(1+s^{2}\right)\right]+\int_{y_{\min }}^{1} d y \mathcal{A}_{y}\left[\frac{3 s^{4} x^{2}\left(y^{2}-1\right)}{1+s^{2}}-\frac{9 s^{4}}{2}\right. \\
& \left.+10 x y\left(3 \arctan s-3 s+s^{3}\right)+\left(9+6 u^{2}-6 x^{2} y^{2}\right)\left[s^{2}-\ln \left(1+s^{2}\right)\right]\right],
\end{aligned}
$$

with $x=p / m_{\pi}$ and the auxiliary functions $y_{\min }=\sqrt{1-u^{2} / x^{2}}$ and $s=x y+\sqrt{u^{2}-x^{2}+x^{2} y^{2}}$. In order to keep the notation compact we have introduced the antisymmetrization prescription $\mathcal{A}_{y}[f(y)]=f(y)-f(-y)$. Note that there is a term in eq.(15) which vanishes identically above $p=\sqrt{2} k_{f}$. A geometrical explanation for this non-smooth behavior is that an orthogonal pair of vectors connecting the origin with two points inside a sphere ceases to exist if the center of the sphere is displaced too far from the origin (namely by more than $\sqrt{2}$ times the sphere radius). The orthogonality of the (momentum difference) vectors is imposed here by the nonrelativistic on-mass-shell condition for a nucleon. The combined two-body, three-body and four-body contributions to $W\left(p, k_{f}\right)$ from the iterated $1 \pi$-exchange Fock diagram read on the other hand (for $p>k_{f}$ ):

$$
\begin{aligned}
W\left(p, k_{f}\right)^{(F)=} & \frac{\pi g_{A}^{4} M m_{\pi}^{4}}{\left(4 \pi f_{\pi}\right)^{4}}\left\{u^{3} x+\frac{u^{5}}{5 x}+\frac{3}{2 x} \int_{(x-u) / 2}^{(x+u) / 2} d \xi\left[(2 \xi-x)^{2}-u^{2}\right] \frac{1+4 \xi^{2}}{1+2 \xi^{2}} \ln \left(1+4 \xi^{2}\right)\right. \\
& +\frac{3}{4 \pi} \theta(\sqrt{2} u-x) \int_{y_{\min }}^{1} d y \int_{y_{\min }}^{1} d z \frac{\theta\left(1-y^{2}-z^{2}\right)}{\sqrt{1-y^{2}-z^{2}}} \mathcal{A}_{y}\left[s^{2}-\ln \left(1+s^{2}\right)\right] \\
& \left.\times \mathcal{A}_{z}\left[t^{2}-\ln \left(1+t^{2}\right)\right]+\frac{3}{x} \int_{-1}^{1} d y \int_{0}^{u} d \xi \xi^{2}\left[\ln \left(1+\sigma^{2}\right)-\sigma^{2}\right]\left(1-\frac{1}{R}\right)\right\},
\end{aligned}
$$

with some new auxiliary functions $t=x z+\sqrt{u^{2}-x^{2}+x^{2} z^{2}}$ and $\sigma=\xi y+\sqrt{u^{2}-\xi^{2}+\xi^{2} y^{2}}$ and $R=\sqrt{\left(1+x^{2}-\xi^{2}\right)^{2}+4 \xi^{2}\left(1-y^{2}\right)}$. It is also interesting to consider the imaginary singleparticle potential $W\left(p, k_{f}\right)$ in the chiral limit $m_{\pi}=0$. One finds the following closed form expressions:

$$
\left.W\left(p, k_{f}\right)\right|_{m_{\pi}=0}=\frac{3 \pi g_{A}^{4} M}{\left(4 \pi f_{\pi}\right)^{4}}\left\{\frac{7 k_{f}^{5}}{5 p}-k_{f}^{3} p-\frac{2}{5 p}\left(2 k_{f}^{2}-p^{2}\right)^{5 / 2} \theta\left(\sqrt{2} k_{f}-p\right)\right\}, \quad p>k_{f},
$$




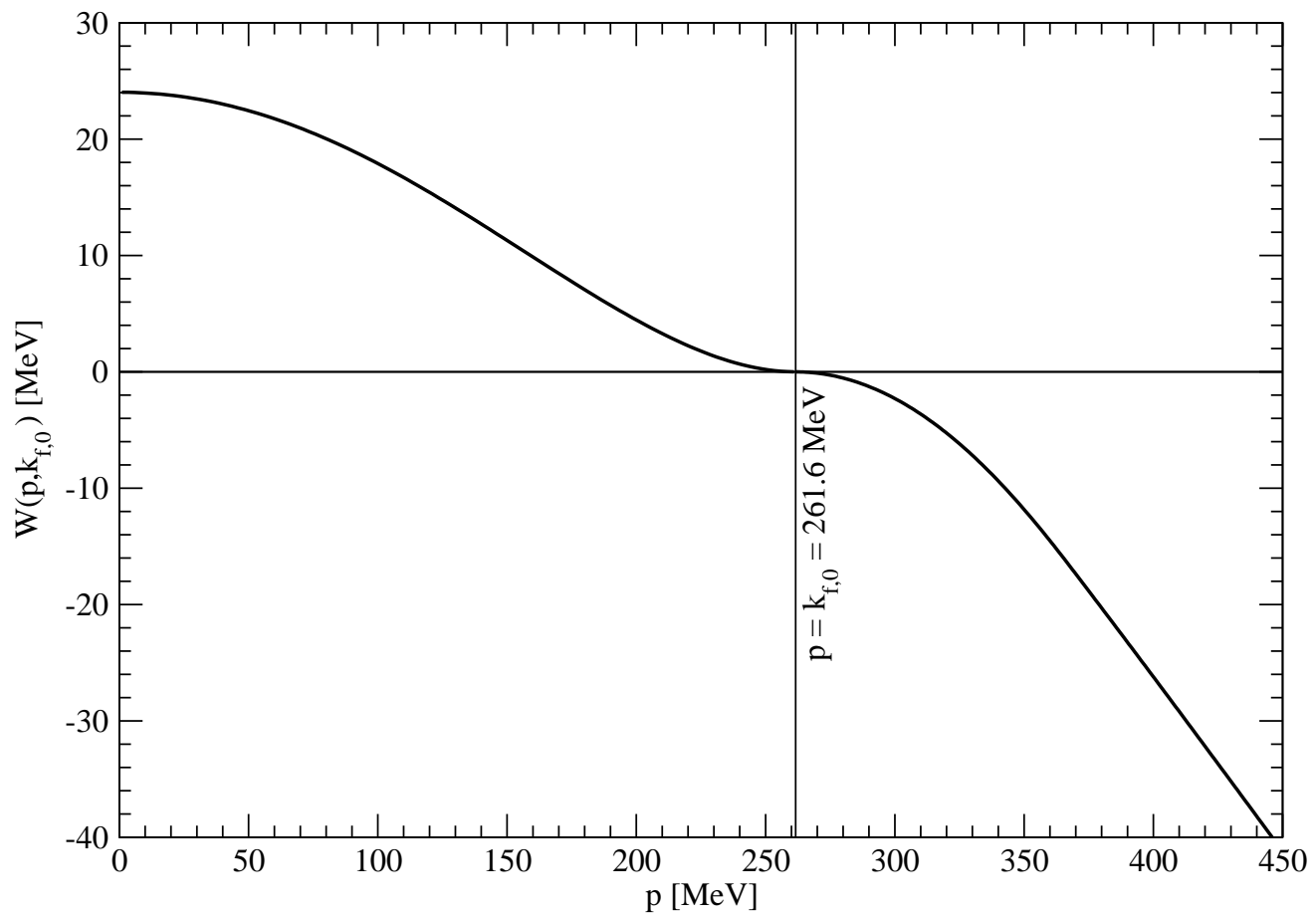

Figure 6: The imaginary part of the single-particle potential $W\left(p, k_{f 0}\right)$ at saturation density $k_{f 0}=261.6 \mathrm{MeV}$ as a function of the nucleon momentum $p$. The quadratic behavior around the Fermi surface $p=k_{f 0}$ with a sign change of the curvature is required by Luttinger's theorem 30].

$$
\left.W\left(p, k_{f}\right)\right|_{m_{\pi}=0}=\frac{9 \pi g_{A}^{4} M}{4\left(4 \pi f_{\pi}\right)^{4}}\left(k_{f}^{2}-p^{2}\right)^{2}, \quad p<k_{f},
$$

to which the iterated $1 \pi$-exchange Hartree and Fock diagrams have contributed in the ratio $4:-1$. The analytical results in eqs. $(17,18)$ agree with Galitskii's calculation [29] of a contactinteraction to second order. In the chiral limit $m_{\pi}=0$ the spin-averaged product of two $\pi N N$ interaction vertices gets canceled by the pion propagators and thus one is effectively dealing with a zero-range NN-contact interaction at second order. The agreement with Galitskii's result 29 serves as an important check on the technically involved calculation behind eqs. $(15,16)$.

Fig. 6 shows the imaginary part of the single-particle potential $W\left(p, k_{f 0}\right)$ at saturation density $k_{f 0}=261.6 \mathrm{MeV}$ as a function of the nucleon momentum $p$. The quantity $\pm 2 W\left(p, k_{f}\right)$ determines the width of a hole-state or a particle-state of momentum $p<k_{f}$ or $p>k_{f}$. The finite life time of these states originates from redistributing energy into additional particle-hole excitations. Our predicted value $W\left(0, k_{f 0}\right)=24.0 \mathrm{MeV}$ at $p=0$ lies in between the results $W\left(0, k_{f 0}\right) \simeq 20 \mathrm{MeV}$ of ref. 31] employing the Gogny D1 effective interaction and $W\left(0, k_{f 0}\right) \simeq$ $40 \mathrm{MeV}$ of ref. 24] using the Paris NN-potential. As a consequence of the decreasing phasespace for redistributing the hole-state energy, the curve in Fig. 6 drops with momentum $p$ and $W\left(p, k_{f 0}\right)$ reaches zero at the Fermi surface $p=k_{f 0}$. According to Luttinger's theorem [30] this vanishing is of quadratic order $\sim\left(p-k_{f}\right)^{2}$, a feature which is clearly exhibited by the curve in Fig. 6.

When crossing the Fermi surface the curvature of the imaginary single-particle potential $W\left(p, k_{f}\right)$ flips the sign. From there on a rapid fall to negative values sets in. In fact the width $\Gamma_{s p}=-2 W\left(p, k_{f}\right)$ represents the spreading of a single-particle state above the Fermi surface into two-particle-one-hole states with growing phase space as $p-k_{f}$ increases. The range of validity 
of the present chiral perturbation theory calculation is again expected to be $p<400 \mathrm{MeV}$. The rapid growth of $\Gamma_{s p}$ beyond such limited momentum scales is not shared by the results of refs. [24, 31].

\section{$5 \quad$ Nuclear energy density functional}

The energy density functional is a general starting point for (non-relativistic) nuclear structure calculations within the framework of the self-consistent mean-field approximation [32]. In this context effective Skyrme forces [33, 34, 35] have gained much popularity because of their analytical simplicity and their ability to reproduce nuclear properties over the whole periodic table. In a recent work [36] we have calculated the nuclear energy density functional which emerges from (leading and next-to-leading order) chiral pion-nucleon dynamics. The calculation in ref. 36] included (only) the $1 \pi$-exchange Fock-diagram and the iterated $1 \pi$-exchange Hartree and Fock diagrams. These few components alone already lead to a good nuclear matter equation of state $\bar{E}\left(k_{f}\right)$. Therefore the interest here is on the additional effects from $2 \pi$-exchange with virtual $\Delta$-excitation contributing one order higher in the small momentum expansion. Going up to quadratic order in spatial gradients (i.e. deviations from homogeneity) the energy density functional relevant for $\mathrm{N}=\mathrm{Z}$ even-even nuclei reads [36]:

$$
\mathcal{E}[\rho, \tau]=\rho \bar{E}\left(k_{f}\right)+\left[\tau-\frac{3}{5} \rho k_{f}^{2}\right]\left[\frac{1}{2 M}-\frac{5 k_{f}^{2}}{56 M^{3}}+F_{\tau}\left(k_{f}\right)\right]+(\vec{\nabla} \rho)^{2} F_{\nabla}\left(k_{f}\right),
$$

with $\rho(\vec{r})=2 k_{f}^{3}(\vec{r}) / 3 \pi^{2}$ the local nucleon density (expressed in terms of a local Fermi momentum $\left.k_{f}(\vec{r})\right)$ and $\tau(\vec{r})$ the local kinetic energy density. We have left out in eq.(19) the spin-orbit coupling term since the corresponding results (for $\Delta$-driven $2 \pi$-exchange three-body spin-orbit forces $^{3}$ ) can be found in ref. [37]. In phenomenological Skyrme parameterizations, the strength function $F_{\tau}\left(k_{f}\right)$ goes linearly with the density $\rho$, while $F_{\nabla}\left(k_{f}\right)$ is constant. The starting point for the construction of an explicit nuclear energy density functional $\mathcal{E}[\rho, \tau]$ is the bilocal densitymatrix as given by a sum over the occupied energy eigenfunctions: $\sum_{\alpha \in \text { occ }} \Psi_{\alpha}(\vec{r}-\vec{a} / 2) \Psi_{\alpha}^{\dagger}(\vec{r}+$ $\vec{a} / 2$ ). According to Negele and Vautherin [38] it can be expanded in relative and center-of-mass coordinate, $\vec{a}$ and $\vec{r}$, with expansion coefficients determined by purely local quantities (nucleon density, kinetic energy density and spin-orbit density). As outlined in section 2 of ref. 36] the Fourier transform of the (so-expanded) density matrix defines in momentum space a "medium insertion" for the inhomogeneous many-nucleon system:

$$
\Gamma(\vec{p}, \vec{q})=\int d^{3} r e^{-i \vec{q} \cdot \vec{r}} \theta\left(k_{f}-|\vec{p}|\right)\left\{1+\frac{35 \pi^{2}}{8 k_{f}^{7}}\left(5 \vec{p}^{2}-3 k_{f}^{2}\right)\left[\tau-\frac{3}{5} \rho k_{f}^{2}-\frac{1}{4} \vec{\nabla}^{2} \rho\right]\right\} .
$$

The strength function $F_{\tau}\left(k_{f}\right)$ in eq.(19) emerges via a perturbation on top of the density of states $\theta\left(k_{f}-|\vec{p}|\right)$. As a consequence of that, $F_{\tau}\left(k_{f}\right)$ can be directly expressed in terms of the real single-particle potential $U\left(p, k_{f}\right)$ as:

$$
F_{\tau}\left(k_{f}\right)=\frac{35}{4 k_{f}^{7}} \int_{0}^{k_{f}} d p p^{2}\left(5 p^{2}-3 k_{f}^{2}\right) U\left(p, k_{f}\right)
$$

Note that any $p$-independent contribution, in particular the $B_{3}$-term in eq.(9) and the $\zeta$-term in eq.(11), drops out. In the medium insertion eq.(20) $\tau-3 \rho k_{f}^{2} / 5$ is accompanied by $-\vec{\nabla}^{2} \rho / 4$.

\footnotetext{
${ }^{3}$ Interestingly, this three-body spin-orbit coupling is not a relativistic effect but independent of the nucleon mass $M$.
} 
After performing a partial integration one is lead to the following decomposition:

$$
F_{\nabla}\left(k_{f}\right)=\frac{\pi^{2}}{8 k_{f}^{2}} \frac{\partial F_{\tau}\left(k_{f}\right)}{\partial k_{f}}+F_{d}\left(k_{f}\right),
$$

where $F_{d}\left(k_{f}\right)$ comprises all those contributions for which the $(\vec{\nabla} \rho)^{2}$-factor originates directly from the momentum-dependence of the interactions.

We enumerate now the contributions to the strength functions $F_{\tau, d}\left(k_{f}\right)$ generated by $2 \pi$ exchange with virtual $\Delta$-excitation. We start with (regularization dependent) contributions encoded in subtraction constants:

$$
F_{\tau}\left(k_{f}\right)^{(c t)}=B_{5} \frac{5 k_{f}^{3}}{3 M^{4}}, \quad F_{d}\left(k_{f}\right)^{(c t)}=\frac{B_{d}}{M^{4}},
$$

where the new parameter $B_{d}=-M^{4} V_{C}^{\prime \prime}(0) / 4$ stems from two-body Hartree diagrams and the momentum transfer dependence of the isoscalar central NN-amplitude $V_{C}(q)$. Two-body Fock diagrams contribute only to $F_{\tau}\left(k_{f}\right)$ via a (subtracted) dispersion integral:

$$
\begin{aligned}
F_{\tau}\left(k_{f}\right)^{(2 F)}= & \frac{35}{24 \pi^{3} k_{f}^{4}} \int_{2 m_{\pi}}^{\infty} d \mu \operatorname{Im}\left(V_{C}+3 W_{C}+2 \mu^{2} V_{T}+6 \mu^{2} W_{T}\right)\left\{\frac{8 k_{f}^{7}}{35 \mu^{3}}-\frac{\mu k_{f}^{3}}{3}-6 \mu^{3} k_{f}\right. \\
& \left.+\frac{\mu^{5}}{4 k_{f}}+5 \mu^{4} \arctan \frac{2 k_{f}}{\mu}+\frac{\mu^{3}}{16 k_{f}^{3}}\left(24 k_{f}^{4}-18 k_{f}^{2} \mu^{2}-\mu^{4}\right) \ln \left(1+\frac{4 k_{f}^{2}}{\mu^{2}}\right)\right\} .
\end{aligned}
$$

The evaluation of the (left) three-body Hartree diagram in Fig. 2 leads to the results:

$$
\begin{gathered}
F_{\tau}\left(k_{f}\right)^{(3 H)}=\frac{35 g_{A}^{4} m_{\pi}^{4}}{\Delta\left(2 \pi f_{\pi}\right)^{4}}\left\{\frac{13}{4}-\frac{5}{24 u^{2}}+\frac{u^{2}}{9}-\frac{35}{12 u} \arctan 2 u+\left(\frac{5}{96 u^{4}}+\frac{3}{4 u^{2}}-\frac{3}{4}\right) \ln \left(1+4 u^{2}\right)\right\}, \\
F_{d}\left(k_{f}\right)^{(3 H)}=\frac{g_{A}^{4} m_{\pi}}{128 \pi^{2} \Delta f_{\pi}^{4}}\left\{23 \arctan 2 u-\frac{7}{u} \ln \left(1+4 u^{2}\right)-16 u-\frac{2 u\left(3+16 u^{2}\right)}{3\left(1+4 u^{2}\right)^{2}}\right\} .
\end{gathered}
$$

Somewhat more involved is the evaluation of the (right) Fock diagram in Fig. 2 for which we find:

$$
\begin{aligned}
F_{\tau}\left(k_{f}\right)^{(3 F)=} & \frac{35 g_{A}^{4} m_{\pi}^{4}}{\Delta\left(8 \pi f_{\pi}\right)^{4} u^{7}} \int_{0}^{u} d x\left[2 G_{S}(x, u) \widetilde{G}_{S}(x, u)+G_{T}(x, u) \widetilde{G}_{T}(x, u)\right], \\
\widetilde{G}_{S}(x, u)= & \frac{4 u x}{3}\left(6 u^{4}-22 u^{2}-45+30 x^{2}-10 u^{2} x^{2}\right) \\
+ & 4 x\left(10+9 u^{2}-15 x^{2}\right)[\arctan (u+x)+\arctan (u-x)] \\
+ & \left(35 x^{2}+14 u^{2} x^{2}-10 x^{4}-5-9 u^{2}-4 u^{4}\right) \ln \frac{1+(u+x)^{2}}{1+(u-x)^{2}} \\
\widetilde{G}_{T}(x, u)= & \frac{u x}{12}\left(69 u^{4}+70 u^{2}-15\right)-\frac{u x^{3}}{12}\left(45+31 u^{2}\right)-\frac{15 u x^{5}}{4} \\
& \quad-\frac{u}{4 x}\left(1+u^{2}\right)^{2}\left(5+3 u^{2}\right)+\frac{\left[1+(u+x)^{2}\right]\left[1+(u-x)^{2}\right]}{16 x^{2}} \\
& \quad \times\left(5+8 u^{2}+3 u^{4}-18 u^{2} x^{2}+15 x^{4}\right) \ln \frac{1+(u+x)^{2}}{1+(u-x)^{2}}
\end{aligned}
$$




$$
\begin{aligned}
F_{d}\left(k_{f}\right)^{(3 F)=} & \frac{g_{A}^{4} m_{\pi}}{\pi^{2} \Delta\left(8 f_{\pi}\right)^{4}}\left\{-\frac{3+12 u^{2}+26 u^{4}+40 u^{6}}{u^{5}\left(1+4 u^{2}\right)} \ln \left(1+4 u^{2}\right)\right. \\
& \left.+\frac{3}{8 u^{7}}\left(1+2 u^{2}+8 u^{4}\right) \ln ^{2}\left(1+4 u^{2}\right)+\frac{2\left(3+6 u^{2}+16 u^{4}\right)}{u^{3}\left(1+4 u^{2}\right)}\right\},
\end{aligned}
$$

with $G_{S, T}(x, u)$ defined in eqs. $(7,8)$. The strength functions $F_{\tau, d}\left(k_{f}\right)$ are completed by adding to the terms in eqs. (23-30) the contributions from $1 \pi$-exchange and iterated $1 \pi$-exchange written down in eqs. $(9,11,12,14,15,18,19,22,24,27,28)$ of ref. [36]. In order to be consistent with the calculation of the energy per particle $\bar{E}\left(k_{f}\right)$ and the single-particle potential $U\left(p, k_{f}\right)$ we complete the $1 \pi$-exchange contribution by its relativistic $1 / M^{2}$-correction:

$$
\begin{aligned}
F_{\tau}\left(k_{f}\right)^{(1 \pi)}= & \frac{g_{A}^{2} m_{\pi}^{3} u^{-5}}{\left(32 \pi f_{\pi} M\right)^{2}}\left\{\frac{280}{3} u^{6}-\frac{15}{2}+2 u\left(525-700 u^{2}-96 u^{4}\right) \arctan 2 u\right. \\
& \left.-64 u^{8}+744 u^{4}-1777 u^{2}+\left(1050 u^{2}-77+\frac{15}{8 u^{2}}\right) \ln \left(1+4 u^{2}\right)\right\} .
\end{aligned}
$$

The expression in eq.(19) multiplying the kinetic energy density $\tau(\vec{r})$ has the interpretation of a reciprocal density dependent effective nucleon mass:

$$
\widetilde{M}^{*}(\rho)=M\left[1-\frac{5 k_{f}^{2}}{28 M^{2}}+2 M F_{\tau}\left(k_{f}\right)\right]^{-1} .
$$

We note that this effective nucleon mass $\widetilde{M}^{*}(\rho)$ (entering the nuclear energy density functional) is conceptually different from the so-called "Landau"-mass $M^{*}\left(k_{f}\right)$ defined in eq.(13). Only if the real single-particle potential has a simple quadratic dependence on the nucleon momentum, $U\left(p, k_{f}\right)=U_{0}\left(k_{f}\right)+p^{2} U_{1}\left(k_{f}\right)$, do these two variants of effective nucleon mass agree with each other (modulo very small differences related to the relativistic $\left(k_{f} / 2 M\right)^{2}$-correction).

In Fig. 7 we show the ratio effective-over-free nucleon mass $\widetilde{M}^{*}(\rho) / M$ as a function of the nucleon density $\rho=2 k_{f}^{3} / 3 \pi^{2}$. The dotted line corresponds to the result of ref. [36] based on $1 \pi$ - and iterated $1 \pi$-exchange only. The full line includes in addition the effects from $2 \pi$ exchange with virtual $\Delta$-excitation. It is clearly visible that the inclusion of the $\pi N \Delta$-dynamics leads to substantial improvement of the effective nucleon mass $\widetilde{M}^{*}(\rho)$ since now it decreases monotonically with the density. This behavior is of course a direct reflection of the improved momentum dependence of the real single-particle potential $U\left(p, k_{f}\right)$ (see Fig. 4). Our prediction for the effective nucleon mass at saturation density, $\widetilde{M}^{*}\left(\rho_{0}\right)=0.64 M$, is comparable to the typical value $\widetilde{M}^{*}\left(\rho_{0}\right) \simeq 0.7 M$ of phenomenological Skyrme forces [33, 34]. The full curve in Fig. 7 displays another interesting and important feature, namely strong curvature effects at low densities $\rho<0.05 \mathrm{fm}^{-3}$. They originate from the explicit presence of the small mass scale $m_{\pi}=135 \mathrm{MeV}$ in our calculation.

Fig. 8 shows the strength function $F_{\nabla}\left(k_{f}\right)$ belonging to the $(\vec{\nabla} \rho)^{2}$-term in the nuclear energy density functional versus the nucleon density $\rho=2 k_{f}^{3} / 3 \pi^{2}$. The dotted line gives the result of ref. [36] based on $1 \pi$ - and iterated $1 \pi$-exchange only and the full line includes in addition the effects from $2 \pi$-exchange with virtual $\Delta$-excitation. The subtraction constant $B_{d}$ (representing density independent short-range contributions) has been set to zero. In the region around saturation density $\rho_{0} \simeq 0.16 \mathrm{fm}^{-3}$ one observes a clear improvement since there the full line meets the band spanned by the three phenomenological Skyrme forces SIII [33], Sly 34] and MSk [35. The strong rise of $F_{\nabla}\left(k_{f}\right)$ towards low densities remains however. As explained in ref. [36] this has to do with chiral singularities (of the form $m_{\pi}^{-2}$ and $m_{\pi}^{-1}$ ) in the contributions from $1 \pi$-exchange and iterated $1 \pi$-exchange. 


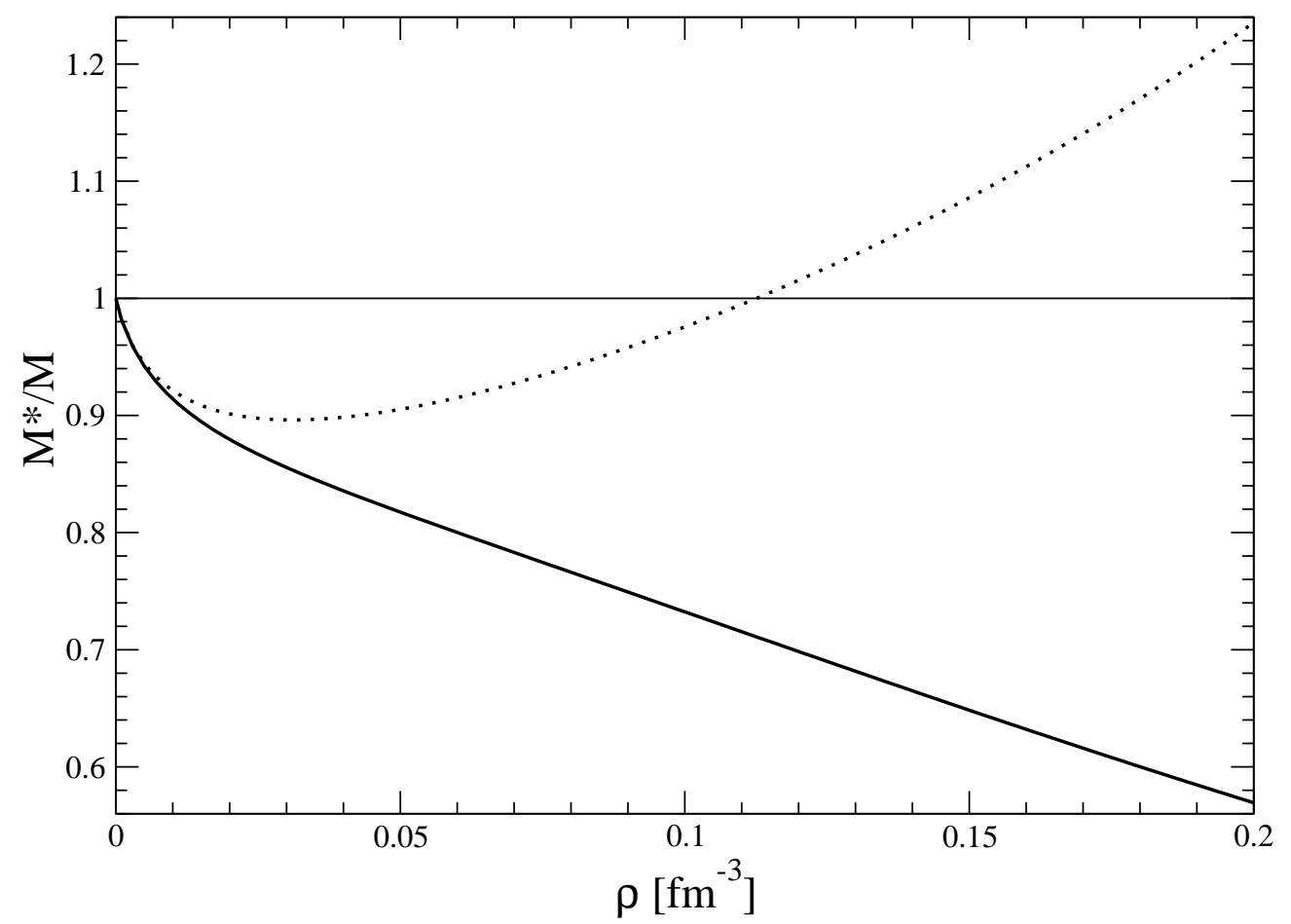

Figure 7: The effective nucleon mass $\widetilde{M}^{*}(\rho)$ divided by the free nucleon mass $M$ as a function of the nucleon density $\rho$. The dotted line shows the result of ref. [36] based on single and iterated pion-exchange only. The full line includes in addition the effects from $2 \pi$-exchange with virtual $\Delta$-excitation.

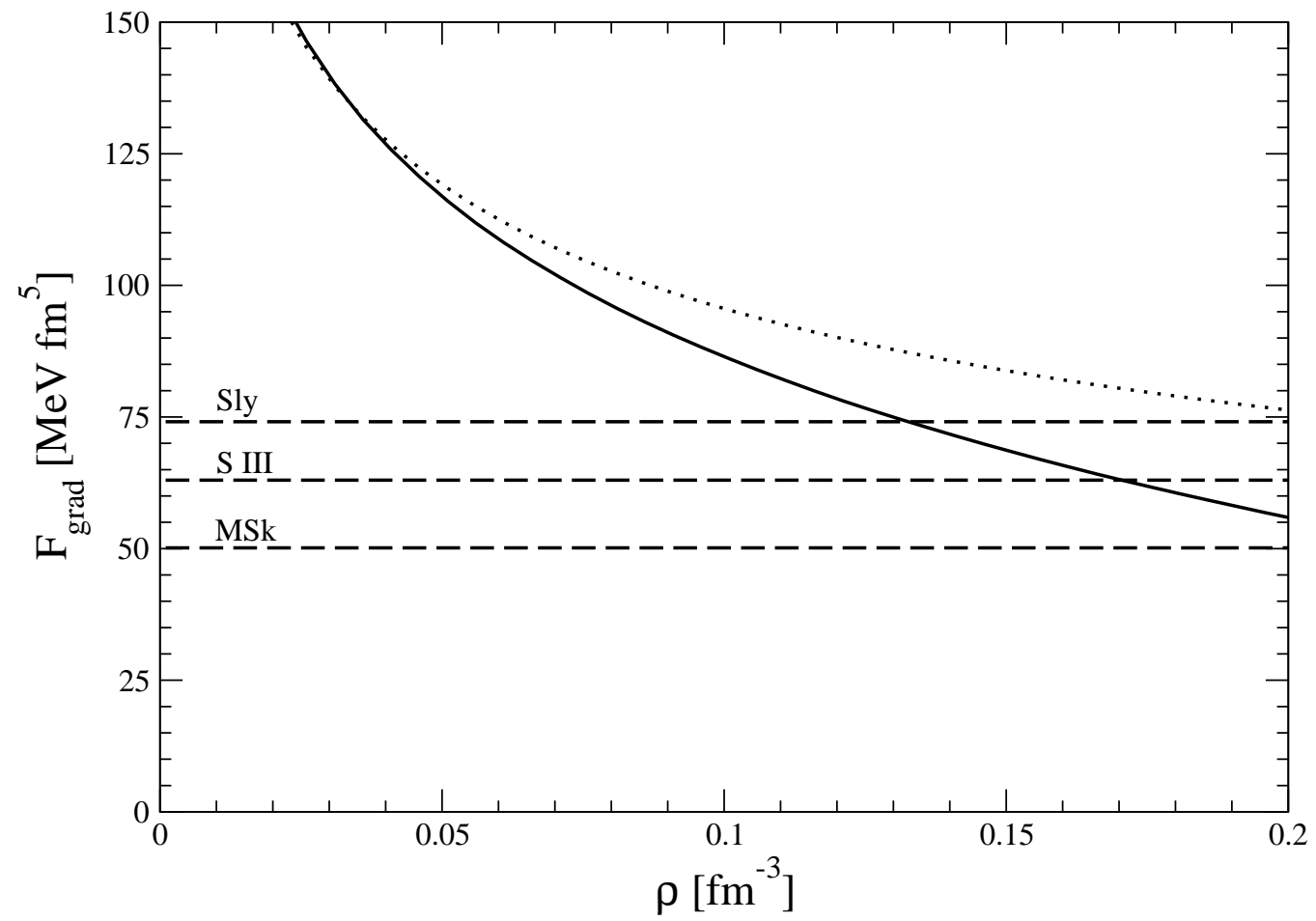

Figure 8: The strength function $F_{\nabla}\left(k_{f}\right)$ multiplying the $(\vec{\nabla} \rho)^{2}$-term in the nuclear energy density functional versus the nucleon density $\rho=2 k_{f}^{3} / 3 \pi^{2}$. The dotted line shows the result of ref. 36 based on single and iterated pion-exchange only. The full line includes in addition the effects from $2 \pi$-exchange with virtual $\Delta$-excitation. 
The knowledge of the strength function $F_{\nabla}\left(k_{f}\right)$ and the equation of state $\bar{E}\left(k_{f}\right)$ allows one to calculate the surface energy of semi-infinite nuclear matter [39] via:

$$
a_{s}=2\left(36 \pi \rho_{0}^{-2}\right)^{1 / 3} \int_{0}^{\rho_{0}} d \rho \sqrt{\rho F_{\nabla}\left(k_{f}\right)\left[\bar{E}\left(k_{f}\right)-\bar{E}_{0}\right]} .
$$

Here, we have inserted into the formula for the surface energy $a_{s}$ (see eq.(5.32) in ref. 39]) that density profile $\rho(z)$ which minimizes it. Numerical evaluation of eq.(33) gives $a_{s}=24.2 \mathrm{MeV}$. This number overestimates semi-empirical determinations of the surface energy, such as $a_{s}=$ $20.7 \mathrm{MeV}$ of ref. [4] or $a_{s}=18.2 \mathrm{MeV}$ of ref. [39], by $17 \%$ or more. ${ }^{4}$ The reason for our high value $a_{s}=24.2 \mathrm{MeV}$ is of course the strong rise of the strength function $F_{\nabla}\left(k_{f}\right)$ at low densities. Its derivation is based on the density-matrix expansion of Negele and Vautherin [38] which has been found to become inaccurate at low and non-uniform densities [41]. Therefore one should not trust the curves in Fig. 8 below $\rho=0.05 \mathrm{fm}^{-3}$. Getting the right order of magnitude for $F_{\nabla}\left(k_{f}\right)$ in the density region $0.1 \mathrm{fm}^{-3}<\rho<0.2 \mathrm{fm}^{-3}$ is already a satisfactory result.

\section{$6 \quad$ Nuclear matter at finite temperatures}

In this section, we discuss nuclear matter at finite temperatures $T \leq 30 \mathrm{MeV}$. We are particularly interested in the first-order liquid-gas phase transition of isospin-symmetric nuclear matter and its associated critical point $\left(\rho_{c}, T_{c}\right)$. As outlined in ref. [6] a thermodynamically consistent extension of the present (perturbative) calculational scheme to finite temperatures is to relate it directly to the free energy per particle $\bar{F}(\rho, T)$, whose natural thermodynamical variables are the nucleon density $\rho$ and the temperature $T$. In that case the free energy density $\rho \bar{F}(\rho, T)$ of isospin-symmetric nuclear matter consists of a sum of convolution integrals over interaction kernels $\mathcal{K}_{j}$ multiplied by powers of the density of nucleon states in momentum space: ${ }^{5}$

$$
d\left(p_{j}\right)=\frac{p_{j}}{2 \pi^{2}}\left[1+\exp \frac{p_{j}^{2}-2 M \tilde{\mu}}{2 M T}\right]^{-1}
$$

The effective one-body "chemical potential" $\tilde{\mu}(\rho, T)$ entering the Fermi-Dirac distribution in eq.(34) is determined by the relation to the particle density $\rho=4 \int_{0}^{\infty} d p_{1} p_{1} d\left(p_{1}\right)$. We summarize now the additional interaction kernels arising from $2 \pi$-exchange with virtual $\Delta$-excitation. The two-body kernels read:

$$
\begin{aligned}
& \mathcal{K}_{2}^{(c t)}=24 \pi^{2} B_{3} \frac{p_{1} p_{2}}{M^{2}}+20 \pi^{2} B_{5} \frac{p_{1} p_{2}}{M^{4}}\left(p_{1}^{2}+p_{2}^{2}\right), \\
& \mathcal{K}_{2}^{(F)}= \frac{1}{\pi} \int_{2 m_{\pi}}^{\infty} d \mu \operatorname{Im}\left(V_{C}+3 W_{C}+2 \mu^{2} V_{T}+6 \mu^{2} W_{T}\right) \\
& \times\left\{\mu \ln \frac{\mu^{2}+\left(p_{1}+p_{2}\right)^{2}}{\mu^{2}+\left(p_{1}-p_{2}\right)^{2}}-\frac{4 p_{1} p_{2}}{\mu}+\frac{4 p_{1} p_{2}}{\mu^{3}}\left(p_{1}^{2}+p_{2}^{2}\right)\right\} .
\end{aligned}
$$

Note that the $B_{3}$-term in eq. (35) generates a temperature independent contribution to the free energy per particle, $\bar{F}(\rho, T)^{\left(B_{3}\right)}=3 \pi^{2} B_{3} \rho / 2 M^{2}$, which drops linearly with density. Temperature

\footnotetext{
${ }^{4}$ One could reproduce the surface energy $a_{s}=20.7 \mathrm{MeV}$ of ref. [40] by adjusting the short-range parameter $B_{d}$ in eq.(32) to the value $B_{d}=-75$. The full curve for $F_{\nabla}\left(k_{f}\right)$ in Fig. 8 would then be shifted downward by $29 \mathrm{MeVfm}^{5}$. Compared to $B_{3}=-8$ the fitted number $B_{d}=-75$ seems to be rather large.

${ }^{5}$ Since the temperature $T$ is comparable to an average kinetic energy we count $T$ of quadratic order in small momenta.
} 
by the Maxwell construction) terminates at the critical temperature $T_{c}$. From there on the pressure isotherms $P(\rho, T)$ grow monotonically with the nucleon density $\rho$. We find here a critical temperature of $T_{c} \simeq 15 \mathrm{MeV}$ and a critical density of $\rho_{c} \simeq 0.053 \mathrm{fm}^{-3} \simeq \rho_{0} / 3$. This critical temperature is close to the value $T_{c}=(16.6 \pm 0.9) \mathrm{MeV}$ extracted in ref. [42] from experimentally observed limiting temperatures in heavy ion collisions. In comparison, a critical temperature of $T_{c}=(20 \pm 3) \mathrm{MeV}$ has been extracted in ref. 43. from multi-fragmentation data in proton-on-gold collisions. Most other nuclear matter calculations find a critical temperature somewhat higher than our value, typically $T_{c} \simeq 18 \mathrm{MeV}$ 44, 45, 46. The reduction of $T_{c}$ in comparison to $T_{c} \simeq 25.5 \mathrm{MeV}$ obtained previously in ref. [6] results from the much improved momentum dependence of the real single-particle potential $U\left(p, k_{f 0}\right)$ near the Fermi surface $p=k_{f 0}$ (see Fig.4). As a general rule the critical temperature $T_{c}$ grows with the effective nucleon mass $M^{*}\left(k_{f 0}\right)$ at the Fermi surface.

The single-particle properties around the Fermi surface are decisive for the thermal excitations and therefore they crucially influence the low temperature behavior of nuclear matter. The inclusion of the chiral $\pi N \Delta$-dynamics leads to a realistic value of the density of (thermally excitable) nucleon states at the Fermi surface. This is an important observation.

\section{$7 \quad$ Equation of state of pure neutron matter}

This section is devoted to the equation of state of pure neutron matter. In comparison to the calculation of isospin-symmetric nuclear matter in section 2 only the isospin factors of the $2 \pi$ exchange diagrams with virtual $\Delta$-excitation change. We summarize now the contributions to the energy per particle $\bar{E}_{n}\left(k_{n}\right)$ of pure neutron matter. The two-body terms read:

$$
\begin{gathered}
\bar{E}_{n}\left(k_{n}\right)^{(c t)}=B_{n, 3} \frac{k_{n}^{3}}{M^{2}}+B_{n, 5} \frac{k_{n}^{5}}{M^{4}} \\
\bar{E}_{n}\left(k_{n}\right)^{(2 F)=} \frac{1}{8 \pi^{3}} \int_{2 m_{\pi}}^{\infty} d \mu \operatorname{Im}\left(V_{C}+W_{C}+2 \mu^{2} V_{T}+2 \mu^{2} W_{T}\right)\left\{3 \mu k_{n}-\frac{4 k_{n}^{3}}{3 \mu}\right. \\
\left.+\frac{8 k_{n}^{5}}{5 \mu^{3}}-\frac{\mu^{3}}{2 k_{n}}-4 \mu^{2} \arctan \frac{2 k_{n}}{\mu}+\frac{\mu^{3}}{8 k_{n}^{3}}\left(12 k_{n}^{2}+\mu^{2}\right) \ln \left(1+\frac{4 k_{n}^{2}}{\mu^{2}}\right)\right\},
\end{gathered}
$$

with $B_{n, 3}$ and $B_{n, 5}$ two new subtraction constants. The relative weights of isoscalar $\left(V_{C, T}\right)$ and isovector $\left(W_{C, T}\right) \mathrm{NN}$-amplitudes have changed by a factor 3 in comparison to eq.(2). The three-body terms generated by the Hartree and Fock diagrams in Fig. 2 read:

$$
\begin{gathered}
\bar{E}_{n}\left(k_{n}\right)^{(3 H)}=\frac{g_{A}^{4} m_{\pi}^{6}}{6 \Delta\left(2 \pi f_{\pi}\right)^{4}}\left[\frac{2}{3} u^{6}+u^{2}-3 u^{4}+5 u^{3} \arctan 2 u-\frac{1}{4}\left(1+9 u^{2}\right) \ln \left(1+4 u^{2}\right)\right] \\
\bar{E}_{n}\left(k_{n}\right)^{(3 F)}=-\frac{g_{A}^{4} m_{\pi}^{6} u^{-3}}{4 \Delta\left(4 \pi f_{\pi}\right)^{4}} \int_{0}^{u} d x\left[G_{S}^{2}(x, u)+2 G_{T}^{2}(x, u)\right]
\end{gathered}
$$

with $G_{S, T}(x, u)$ defined in eqs. $(7,8)$. We emphasize that in this section the meaning of $u$ changes to $u=k_{n} / m_{\pi}$, where $k_{n}$ denotes the neutron Fermi momentum. All three three-body diagrams in Fig. 2 have now the same isospin factor $2 / 3$ since between neutrons only the $2 \pi^{0}$-exchange is possible. Note that the Pauli-exclusion principle forbids a three-neutron contact-interaction and therefore eq.(43) is free of any $\zeta$-term. The additional contributions to $\bar{E}_{n}\left(k_{n}\right)$ from the (relativistically improved) kinetic energy, from $1 \pi$-exchange and from iterated $1 \pi$-exchange have been written down in eqs.(32-37) of ref.[2]. 


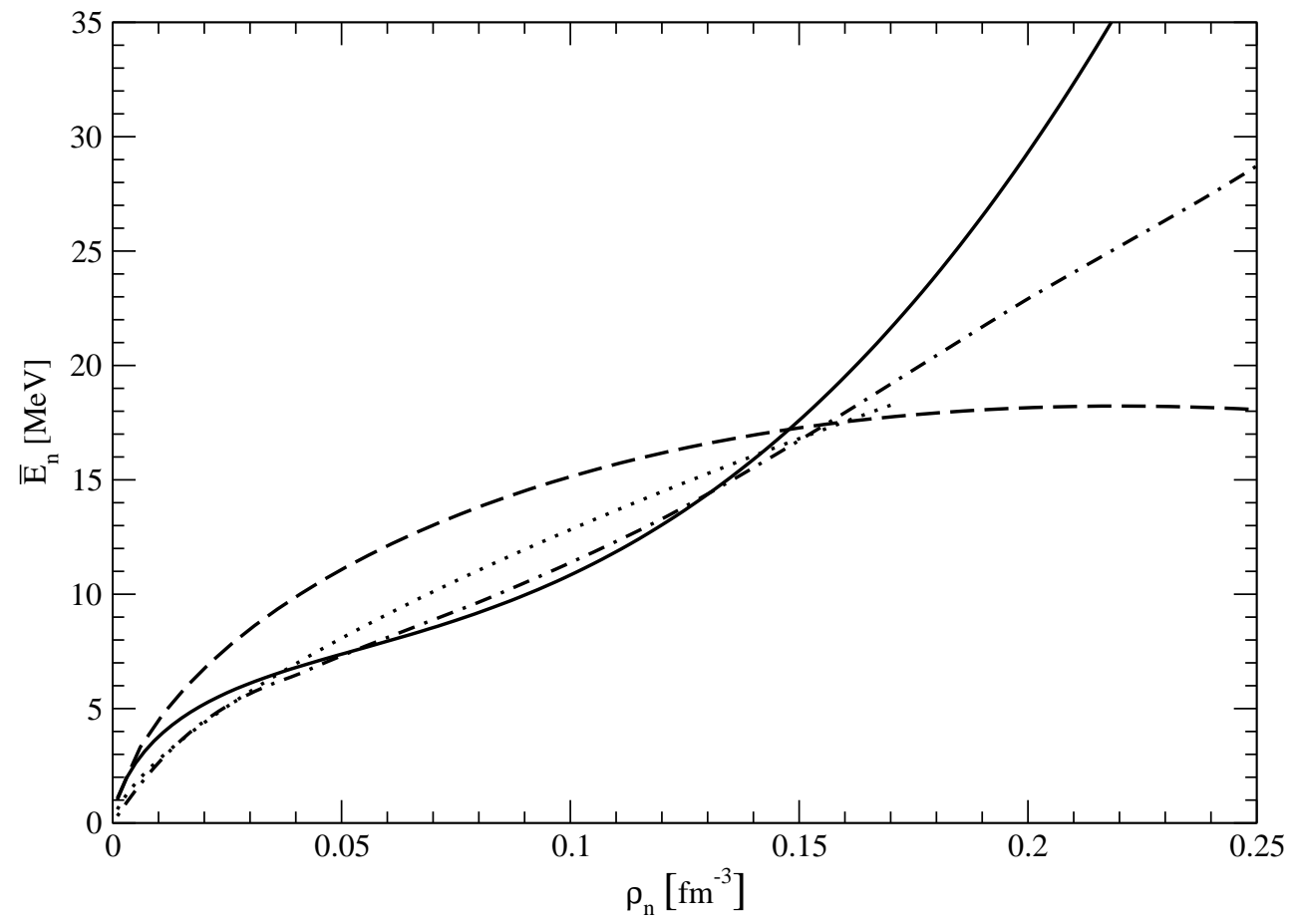

Figure 10: The energy per particle $\bar{E}_{n}\left(k_{n}\right)$ of pure neutron matter as a function of the neutron density $\rho_{n}=k_{n}^{3} / 3 \pi^{2}$. The dashed curve gives the result of ref. [2]. The full curve includes the $\pi N \Delta$-dynamics and two adjusted short-range parameters $B_{n, 3}=-0.94$ and $B_{n, 5}=-3.58$. The dashed-dotted curve stems from the sophisticated many-body calculation of the Urbana group [16]. The dotted curve gives one half of the kinetic energy $\bar{E}_{\text {kin }}\left(k_{n}\right) / 2=3 k_{n}^{2} / 20 M$.

Fig. 10 shows the energy per particle $\bar{E}_{n}\left(k_{n}\right)$ of pure neutron matter as a function of the neutron density $\rho_{n}=k_{n}^{3} / 3 \pi^{2}$. The dashed (concave) curve gives the result of our previous calculation in ref. [2]. The full curve includes the chiral $\pi N \Delta$-dynamics. The short-range parameters $B_{n, 3}$ and $B_{n, 5}$ (controlling the contribution of a nn-contact interaction to $\bar{E}_{n}\left(k_{n}\right)$ ) have been adjusted to the values $B_{n, 3}=-0.95$ and $B_{n, 5}=-3.58 .{ }^{6}$ The dashed-dotted curve in Fig. 10 stems from the sophisticated many-body calculation of the Urbana group [16], to be considered as representative of realistic neutron matter calculations. Moreover, the dotted curve gives one half of the kinetic energy of a free neutron gas, $\bar{E}_{\mathrm{kin}}\left(k_{n}\right) / 2=3 k_{n}^{2} / 20 M$. Results of recent quantum Monte-Carlo calculation in ref. [47] have demonstrated that the neutron matter equation of state at low neutron densities $\rho_{n}<0.05 \mathrm{fm}^{-3}$ is well approximated by this simple form. One observes that up to $\rho_{n}=0.16 \mathrm{fm}^{-3}$ our result for $\bar{E}_{n}\left(k_{n}\right)$ is very close to that of the sophisticated many-body calculation [16, 47]. At higher densities we find a stiffer neutron matter equation of state. Again, one should not expect that our approach works at Fermi momenta larger than $k_{n}=350 \mathrm{MeV}$ (corresponding to $\rho_{n}=0.19 \mathrm{fm}^{-3}$ ). One of the most important results of the present calculation is that the unrealistic downward bending of $\bar{E}_{n}\left(k_{n}\right)$ (as shown by the dashed curve in Fig. 10) disappears after the inclusion of the chiral $\pi N \Delta$-dynamics. This is a manifestation of improved isospin properties.

\footnotetext{
${ }^{6}$ The short-range parameters $B_{n, 3}$ and $B_{n, 5}$ have been adjusted such that the asymmetry energy at saturation density takes on the value $A\left(k_{f 0}\right)=34 \mathrm{MeV}$.
} 
As a further test of isospin properties we consider in this section the density dependent asymmetry energy $A\left(k_{f}\right)$. The asymmetry energy is generally defined by the expansion of the energy per particle of isospin-asymmetric nuclear matter (described by different proton and neutron Fermi momenta $\left.k_{p, n}=k_{f}(1 \mp \delta)^{1 / 3}\right)$ around the symmetry line:

$$
\bar{E}_{\mathrm{as}}\left(k_{p}, k_{n}\right)=\bar{E}\left(k_{f}\right)+\delta^{2} A\left(k_{f}\right)+\mathcal{O}\left(\delta^{4}\right) .
$$

Following the scheme in the previous sections we summarize the contributions to the asymmetry energy $A\left(k_{f}\right)$. The two-body terms read:

$$
\begin{gathered}
A\left(k_{f}\right)^{(c t)}=\left(2 B_{n, 3}-B_{3}\right) \frac{k_{f}^{3}}{M^{2}}+\left(3 B_{n, 5}-B_{5}\right) \frac{10 k_{f}^{5}}{9 M^{4}} \\
A\left(k_{f}\right)^{(2 F)=} \frac{1}{12 \pi^{3}} \int_{2 m_{\pi}}^{\infty} d \mu\left\{\operatorname{Im}\left(V_{C}+2 \mu^{2} V_{T}\right)\left[\mu k_{f}-\frac{2 k_{f}^{3}}{\mu}+\frac{16 k_{f}^{5}}{3 \mu^{3}}-\frac{\mu^{3}}{4 k_{f}} \ln \left(1+\frac{4 k_{f}^{2}}{\mu^{2}}\right)\right]\right. \\
\left.+\operatorname{Im}\left(W_{C}+2 \mu^{2} W_{T}\right)\left[3 \mu k_{f}+\frac{2 k_{f}^{3}}{\mu}-\frac{\mu}{4 k_{f}}\left(8 k_{f}^{2}+3 \mu^{2}\right) \ln \left(1+\frac{4 k_{f}^{2}}{\mu^{2}}\right)\right]\right\} .
\end{gathered}
$$

In eq.(46) we have taken care of the fact that there are only two independent (S-wave) NNcontact couplings which can produce terms linear in density. It is surprising that also the other coefficient $10\left(3 B_{n, 5}-B_{5}\right) / 9$ in front of the $k_{f}^{5} / M^{4}$-term is completely fixed. This fact can be shown on the basis of the most general order- $p^{2}$ NN-contact interaction written down in eq.(6) of ref.48. Out of the seven low-energy constants $C_{1}, \ldots, C_{7}$ only two independent linear combinations, $C_{2}$ and $C_{1}+3 C_{3}+C_{6}$, come into play for homogeneous and spin-saturated nuclear matter. The contribution of the three-body Hartree diagram in Fig. 2 to the asymmetry energy $A\left(k_{f}\right)$ has the following analytical form:

$$
A\left(k_{f}\right)^{(3 H)}=\frac{g_{A}^{4} m_{\pi}^{6} u^{2}}{9 \Delta\left(2 \pi f_{\pi}\right)^{4}}\left[\left(\frac{9}{4}+4 u^{2}\right) \ln \left(1+4 u^{2}\right)-2 u^{4}(1+3 \zeta)-8 u^{2}-\frac{u^{2}}{1+4 u^{2}}\right],
$$

with the abbreviation $u=k_{f} / m_{\pi}$. The parameter $\zeta=-3 / 4$ is again related to the three-nucleon contact interaction $\sim\left(\zeta g_{A}^{4} / \Delta f_{\pi}^{4}\right)(\bar{N} N)^{3}$ which has the interesting property that it contributes equally but with opposite sign to the energy per particle $\bar{E}\left(k_{f}\right)$ and the asymmetry energy $A\left(k_{f}\right)$. Furthermore, both three-body Fock diagrams in Fig. 2 add up to give rise a contribution to the asymmetry $A\left(k_{f}\right)$ which can be represented as:

$$
\begin{aligned}
A\left(k_{f}\right)^{(3 F)=} & \frac{g_{A}^{4} m_{\pi}^{6} u^{-3}}{36 \Delta\left(4 \pi f_{\pi}\right)^{4}} \int_{0}^{u} d x\left\{4 G_{S 01} G_{S 10}-2 G_{S 01}^{2}-6 G_{S 10}^{2}\right. \\
& +2 G_{S}\left(3 G_{S}+8 G_{S 01}-3 G_{S 02}+2 G_{S 11}-3 G_{S 20}\right)+2 G_{T 01} G_{T 10} \\
& \left.-7 G_{T 01}^{2}-3 G_{T 10}^{2}+G_{T}\left(3 G_{T}+8 G_{T 01}-3 G_{T 02}+2 G_{T 11}-3 G_{T 20}\right)\right\} .
\end{aligned}
$$

The auxiliary functions $G_{S, T}(x, u)$ have been defined in eqs. $(7,8)$ and we have introduced a double-index notation for their partial derivatives:

$$
G_{I j k}(x, u)=x^{j} u^{k} \frac{\partial^{j+k} G_{I}(x, u)}{\partial x^{j} \partial u^{k}}, \quad I=S, T, \quad 1 \leq j+k \leq 2 .
$$




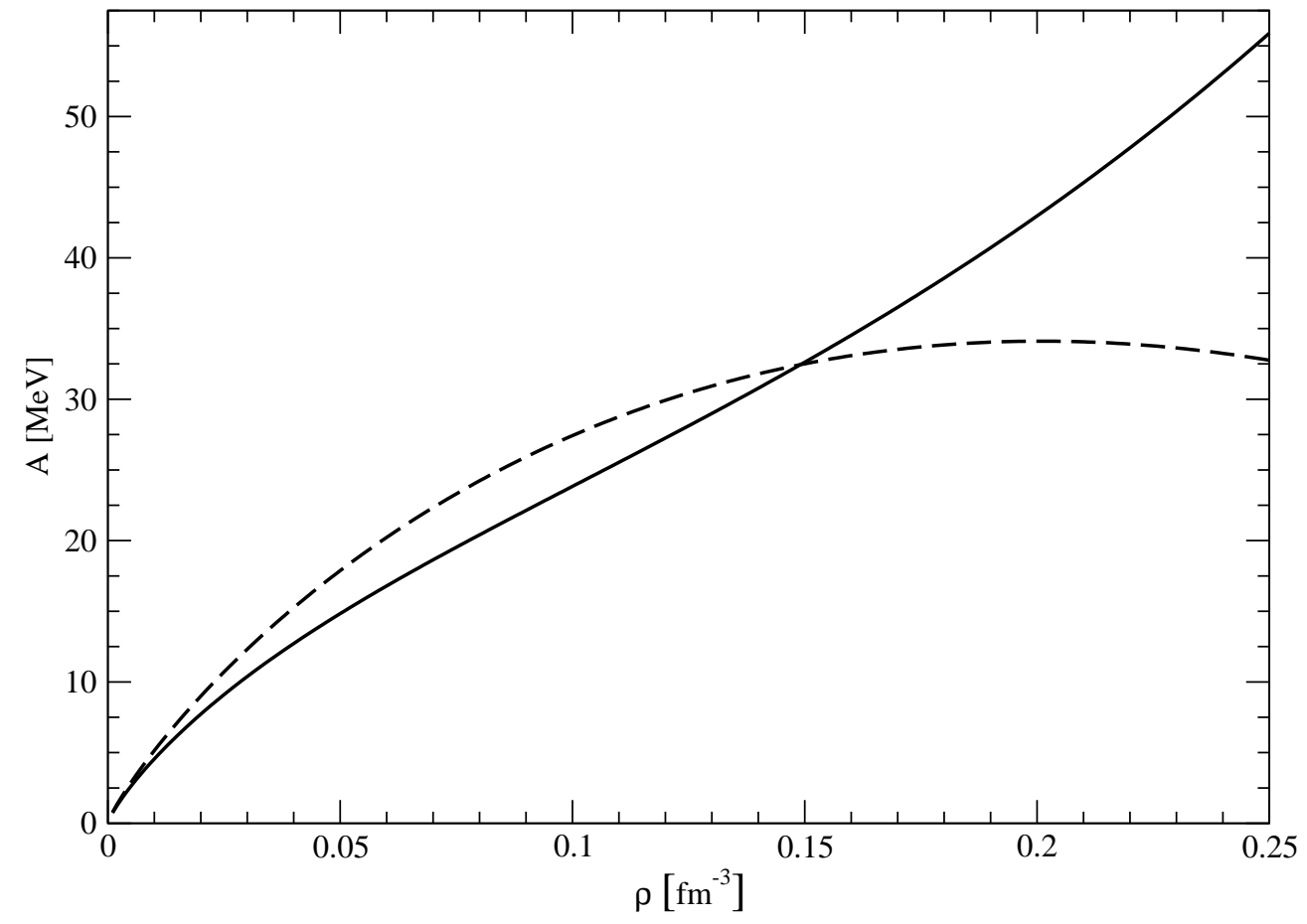

Figure 11: The asymmetry energy $A\left(k_{f}\right)$ as a function of the nucleon density $\rho=2 k_{f}^{3} / 3 \pi^{2}$. The dashed curve shows the result of ref. [2]. The full curve includes the chiral $\pi N \Delta$-dynamics.

For notational simplicity we have omitting the arguments $x$ and $u$ in the integrand of eq.(49). The asymmetry energy $A\left(k_{f}\right)$ is completed by adding to the terms in eqs.(46-49) the contributions from the (relativistically improved) kinetic energy, $1 \pi$-exchange and iterated $1 \pi$-exchange written down in eqs. (20-26) of ref.[2].

In the calculation of the asymmetry energy we use consistently the previously fixed shortdistance parameters $B_{3}=-7.99$ and $B_{n, 3}=-0.95$, as well as $B_{5}=0$ and $B_{n, 5}=-3.58$. Fig. 11 shows the asymmetry energy $A\left(k_{f}\right)$ as a function of the nucleon density $\rho=2 k_{f}^{3} / 3 \pi^{2}$. The dashed (concave) curve corresponds to the result of ref. 2. The full curve includes the chiral $\pi N \Delta$-dynamics. The corresponding value of the asymmetry energy at saturation density $\rho_{0}=0.157 \mathrm{fm}^{-3}$ is $A\left(k_{f 0}\right)=34.0 \mathrm{MeV}$. It decomposes as $A\left(k_{f 0}\right)=(12.1+119.3-109.9+$ 12.5) $\mathrm{MeV}$ into contributions of second, third, fourth and fifth power of small momenta, again with a balance between large third and fourth order terms [2]. The value $A\left(k_{f 0}\right)=34.0 \mathrm{MeV}$ is consistent with most of the existing empirical determinations of the asymmetry energy. For example, a recent microscopic estimate in a relativistic mean-field model (constrained by some specific properties of certain nuclei) gave the value $A\left(k_{f 0}\right)=(34 \pm 2) \mathrm{MeV}$ [49]. For comparison, other empirical values obtained from extensive fits of nuclide masses are $A\left(k_{f 0}\right)=36.8 \mathrm{MeV}$ [40] or $A\left(k_{f 0}\right)=33.2 \mathrm{MeV}$ [50]. The slope of the asymmetry energy at saturation density, $L=$ $k_{f 0} A^{\prime}\left(k_{f 0}\right)$, is likewise an interesting quantity. As demonstrated in Fig. 11 of ref. [51] the neutron skin thickness of ${ }^{208} \mathrm{~Pb}$ is linearly correlated with the slope parameter $L$. We extract from the full curve in Fig. 11 the value $L=90.8 \mathrm{MeV}$. This prediction is not far from $L \simeq 100 \mathrm{MeV}$ quoted in ref. 40] and $L=119.2 \mathrm{MeV}$ obtained from the "standard" relativistic force NL3 [52]. Furthermore, we extract from the curvature of our asymmetry energy $A\left(k_{f}\right)$ at saturation density $\rho_{0}$ the positive asymmetry compressibility $K_{\text {as }}=k_{f 0}^{2} A^{\prime \prime}\left(k_{f 0}\right)-2 L=160.5 \mathrm{MeV}$.

Again, the most important feature visible in Fig. 11 is that the inclusion of the chiral $\pi N \Delta$ dynamics eliminates the (unrealistic) downward bending of the asymmetry $A\left(k_{f}\right)$ at higher densities $\rho>0.2 \mathrm{fm}^{-3}$ (as displayed by the dashed curve in Fig. 11). This is once more a 
manifestation of improved isospin properties.

\section{Isovector single-particle potential}

In this section we generalize the calculation of the single-particle potential to isospin-asymmetric (homogeneous) nuclear matter. Any relative excess of neutrons over protons in the nuclear medium leads to a different "mean-field" for a proton and a neutron. This fact is expressed by the following decomposition of the (real) single-particle potential in isospin-asymmetric nuclear matter:

$$
U\left(p, k_{f}\right)-U_{I}\left(p, k_{f}\right) \tau_{3} \delta+\mathcal{O}\left(\delta^{2}\right), \quad \delta=\frac{\rho_{n}-\rho_{p}}{\rho_{n}+\rho_{p}}
$$

with $U\left(p, k_{f}\right)$ the isoscalar (real) single-particle potential discussed in section 3 . The term linear in the isospin-asymmetry parameter $\delta=\left(\rho_{n}-\rho_{p}\right) /\left(\rho_{n}+\rho_{p}\right)$ defines the (real) isovector singleparticle potential $U_{I}\left(p, k_{f}\right)$, and $\tau_{3} \rightarrow \pm 1$ for a proton or a neutron. Without going into further technical details we summarize now the contributions to $U_{I}\left(p, k_{f}\right)$. The two-body terms read:

$$
\begin{aligned}
U_{I}\left(p, k_{f}\right)^{(c t)}= & \left(2 B_{n, 3}-B_{3}\right) \frac{2 k_{f}^{3}}{M^{2}}+\left(2 B_{n, 5}-B_{5}\right) \frac{5 k_{f}^{3}}{3 M^{4}}\left(k_{f}^{2}+p^{2}\right), \\
U_{I}\left(p, k_{f}\right)^{(2 F)=} & \frac{k_{f}^{2}}{12 \pi^{3}} \int_{2 m_{\pi}}^{\infty} d \mu \operatorname{Im}\left(V_{C}-W_{C}+2 \mu^{2} V_{T}-2 \mu^{2} W_{T}\right) \\
& \times\left\{\frac{4 k_{f}}{\mu^{3}}\left(k_{f}^{2}+p^{2}-\mu^{2}\right)+\frac{\mu}{p} \ln \frac{\mu^{2}+\left(k_{f}+p\right)^{2}}{\mu^{2}+\left(k_{f}-p\right)^{2}}\right\} .
\end{aligned}
$$

The contribution of the three-body Hartree diagram in Fig. 2 and the three-body contact term can be written in analytical form:

$$
U_{I}\left(p, k_{f}\right)^{(3 H)}=\frac{2 g_{A}^{4} m_{\pi}^{6} u^{5}}{9 \Delta\left(2 \pi f_{\pi}\right)^{4}}\left\{\frac{1}{x} \ln \frac{1+(u+x)^{2}}{1+(u-x)^{2}}-\frac{2 u}{\left[1+(u+x)^{2}\right]\left[1+(u-x)^{2}\right]}-2 u(1+3 \zeta)\right\},
$$

and the total contribution of both three-body Fock diagrams in Fig. 2 can be represented as:

$$
\begin{aligned}
U_{I}\left(p, k_{f}\right)^{(3 F)}= & \frac{g_{A}^{4} m_{\pi}^{6} u x^{-2}}{18 \Delta\left(4 \pi f_{\pi}\right)^{4}}\left\{2 G_{S}(x, u) \frac{\partial G_{S}(x, u)}{\partial u}+G_{T}(x, u) \frac{\partial G_{T}(x, u)}{\partial u}\right. \\
& +2 G_{S}(u, u) \frac{\partial G_{S}(u, x)}{\partial x}+G_{T}(u, u) \frac{\partial G_{T}(u, x)}{\partial x} \\
& \left.-\int_{0}^{u} d \xi\left[2 \frac{\partial G_{S}(\xi, u)}{\partial u} \frac{\partial G_{S}(\xi, x)}{\partial x}+7 \frac{\partial G_{T}(\xi, u)}{\partial u} \frac{\partial G_{T}(\xi, x)}{\partial x}\right]\right\},
\end{aligned}
$$

where $x=p / m_{\pi}$ and $u=k_{f} / m_{\pi}$. The auxiliary functions $G_{S, T}(x, u)$ have been defined in eqs. $(7,8)$. The (real) isovector single-particle potential $U_{I}\left(p, k_{f}\right)$ (restricted to the region below the Fermi surface $\left.p \leq k_{f}\right)$ is completed by adding to the terms in eqs.(52-55) the contributions from $1 \pi$-exchange and iterated $1 \pi$-exchange written down in eqs.(28-36) of ref. [53. The imaginary isovector single-particle $W_{I}\left(p, k_{f}\right)$ (below the Fermi surface $p \leq k_{f}$ ) has been discussed in section 4.2 of ref. [53].

The generalization of the Hugenholtz-Van-Hove theorem [21] to isospin-asymmetric nuclear matter gives a model-independent relation for the isovector single-particle potential $U_{I}\left(p, k_{f}\right)$ at the Fermi surface $\left(p=k_{f}\right)$ :

$$
U_{I}\left(k_{f}, k_{f}\right)=2 A\left(k_{f}\right)-\frac{k_{f}^{2}}{3 M}+\frac{k_{f}^{4}}{6 M^{3}}-\left.\frac{k_{f}}{3} \frac{\partial U\left(p, k_{f}\right)}{\partial p}\right|_{p=k_{f}}
$$




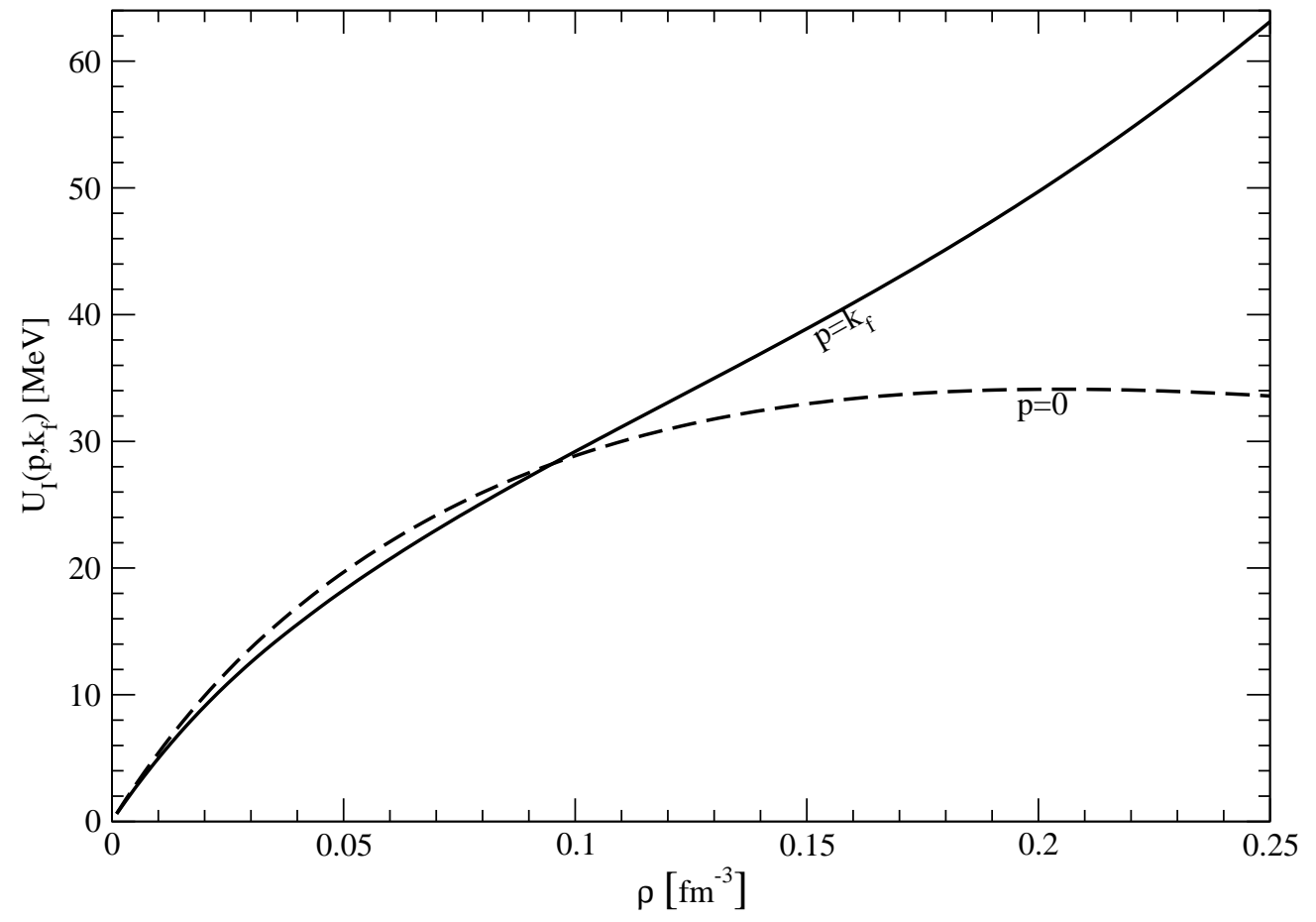

Figure 12: The real isovector single-particle potential $U_{I}\left(p, k_{f}\right)$ as a function of the nucleon density $\rho=2 k_{f}^{3} / 3 \pi^{2}$. The dashed and full curves correspond to the sections $p=0$ (bottom of the Fermi sea) and $p=k_{f}$ (at the Fermi surface), respectively.

The second and third term on the right hand side just subtract non-interacting (kinetic) contributions from the asymmetry energy $A\left(k_{f}\right)$. We find at saturation density $k_{f 0}=261.6 \mathrm{MeV}$ an isovector single-particle potential of $U_{I}\left(k_{f 0}, k_{f 0}\right)=40.4 \mathrm{MeV}$. This is consistent with the value $U_{1} \simeq 40 \mathrm{MeV}[5$ ] deduced from nucleon-nucleus scattering in the framework of the optical model. The generalized Hugenholtz-Van-Hove theorem eq.(56) serves also as an excellent check on our analytical and numerical calculations.

Fig. 12 shows the real isovector single-particle potential $U_{I}\left(p, k_{f}\right)$ as a function of the nucleon density $\rho=2 k_{f}^{3} / 3 \pi^{2}$. The dashed and full curves correspond to the sections $p=0$ (bottom of the Fermi sea) and $p=k_{f}$ (at the Fermi surface), respectively. One observes a splitting of both curves which sets in at $\rho \simeq 0.10 \mathrm{fm}^{-3}$ and increases with the density. At saturation density $\rho_{0}=0.157 \mathrm{fm}^{-3}$ the difference between the (real) isovector single-particle potential at $p=k_{f 0}$ and $p=0$ is $U_{I}\left(k_{f 0}, k_{f 0}\right)-U_{I}\left(0, k_{f 0}\right)=7.1 \mathrm{MeV}$. This is much less than the analogous difference for the (real) isoscalar single-particle potential $U\left(k_{f 0}, k_{f 0}\right)-U\left(0, k_{f 0}\right)=26.4 \mathrm{MeV}$ (see also Fig. 4).

The full line in Fig. 13 shows the (real) isovector single-particle potential $U_{I}\left(p, k_{f 0}\right)$ at saturation density $k_{f 0}=261.6 \mathrm{MeV}$ as a function of the nucleon momentum $p$. In the region below the Fermi surface $p \leq k_{f 0}$ the momentum dependence of this curve is weaker than that of the (real) isoscalar single-particle potential $U\left(p, k_{f 0}\right)$ shown by the left half of the full line in Fig. 4. Finally, we note that the generalized Hugenholtz-Van-Hove theorem eq.(56) (which allows for an alternative determination of $U_{I}\left(k_{f}, k_{f}\right)$ shown in Fig. 12) holds with high numerical precision in our calculation. 


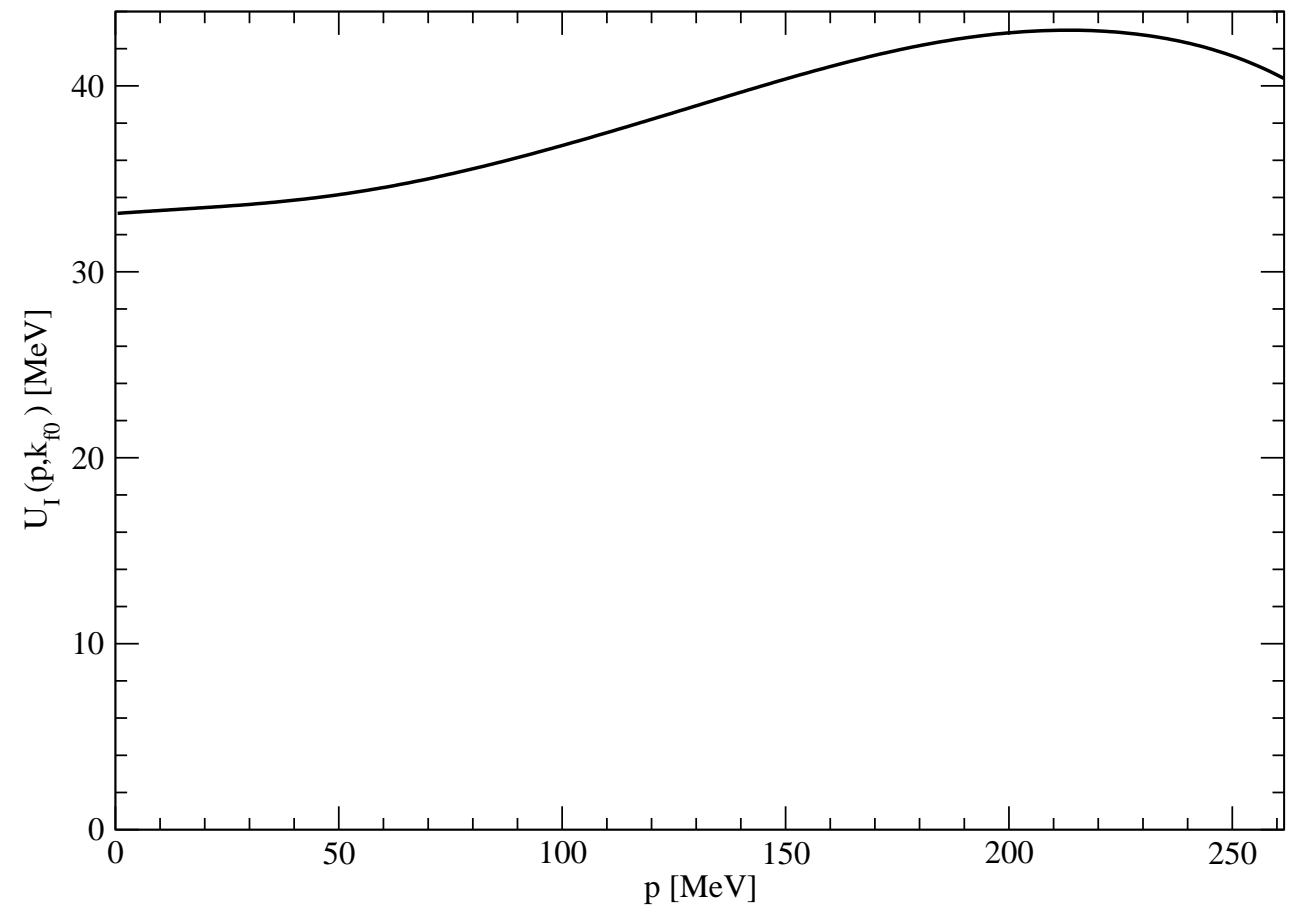

Figure 13: The full line shows the real isovector single-particle potential $U_{I}\left(p, k_{f 0}\right)$ at saturation density $k_{f 0}=261.6 \mathrm{MeV}$ as a function of the nucleon momentum $p$.

\section{Summary and concluding remarks}

In this work we have extended a recent three-loop calculation of nuclear matter in chiral perturbation theory by including the effects from two-pion exchange with single and double virtual $\Delta(1232)$-isobar excitation. In the spirit of an effective field theory, we have encoded all shortdistance contributions (from the high-momentum parts of the pion-loops integrals etc.) in a few adjustable contact-coupling constants. We have investigated a wide variety of nuclear properties in this framework. The empirical saturation point of isospin-symmetric nuclear matter, $\bar{E}_{0}=-16 \mathrm{MeV}, \rho_{0}=0.157 \mathrm{fm}^{-3}$, can be well reproduced by adjusting the strength of a twobody term linear in density and weakening an emerging three-body term quadratic in density. The various constraints set by empirical nuclear matter properties (saturation point and potential depth lead to the (minimal) parameter choice $B_{5}=0$ and $\zeta=-3 / 4$, with little freedom for further variation. The momentum dependence of the real single-particle potential $U\left(p, k_{f}\right)$ is improved significantly by including the chiral $\pi N \Delta$-dynamics. As a consequence the critical temperature of the liquid-gas phase transition gets lowered to the realistic value $T_{c} \simeq 15 \mathrm{MeV}$. The isospin properties of nuclear matter get also substantially improved by including the chiral $\pi N \Delta$-dynamics. The energy per particle of pure neutron matter $\bar{E}_{n}\left(k_{n}\right)$ and the asymmetry energy $A\left(k_{f}\right)$ now show a monotonic growth with density. In the density regime $\rho=2 \rho_{n}<0.2 \mathrm{fm}^{-3}$ relevant for conventional nuclear physics, we find good agreement with sophisticated many-body calculations and (semi)-empirical values.

In passing we note that the inclusion of the chiral $\pi N \Delta$-dynamics guarantees the spinstability of nuclear matter [54]. These improvements can be traced back to repulsive two-body Fock terms as well as three-body terms with a very specific density and momentum dependence. Open questions concerning the role of yet higher orders in the small momentum expansion and its convergence remain and should be further explored.

Our calculation takes seriously the fact that there exist two hadronic scales, the pion mass 
$m_{\pi}=135 \mathrm{MeV}$ and the delta-nucleon mass splitting $\Delta=293 \mathrm{MeV}$, which are smaller than or comparable to the Fermi momentum $k_{f 0} \simeq 262 \mathrm{MeV}$ of equilibrated nuclear matter. Propagation effects of quasi-particles associated with these "light" scales are resolvable. Therefore pions and $\Delta$-isobars must be included as explicit degrees of freedom in the nuclear many-body problem. Controlled by a systematic expansion in small scales $\left(k_{f}, m_{\pi}, \Delta\right)$, the dynamics of the interacting $\pi N \Delta$-system is worked out up to three-loop order. In this effective field theory approach the basic mechanism for nuclear binding and saturation are attractive $2 \pi$-exchange interactions of the van-der-Waals type on which Pauli-blocking acts in the nuclear medium. Most other phenomenological approaches ignore these "light" physical degrees of freedom and parameterize the relevant low-energy dynamics in terms of strongly coupled heavy scalar and vector bosons $(\sigma, \omega, \rho, \delta$, etc.). Their propagation takes place on length scales of $0.5 \mathrm{fm}$ or less and can therefore not be resolved in the domain relevant to nuclear physics. We are guided instead by a change of paradigm, namely that the nuclear many-body problem involves the separation of scales that is characteristic for low-energy QCD and its (chiral) symmetry breaking pattern.

\section{Appendix: Real single-particle potential above the Fermi surface}

In this appendix we summarize the continuation of the real single-particle potential $U\left(p, k_{f}\right)$ calculated in section 3 of ref. 3 into the region above the Fermi surface $p>k_{f}$. The expressions eqs. $(8,9)$ in ref. [3] for the two-body potentials from the $1 \pi$-exchange Fock diagram and the iterated 1 $\pi$-exchange Hartree diagram remain valid without any changes. Eq.(10) in ref. [3] for the two-body potential from the iterated $1 \pi$-exchange Fock diagram gets replaced by:

$$
\begin{aligned}
U_{2}\left(p, k_{f}\right)= & \frac{g_{A}^{4} M m_{\pi}^{4}}{(4 \pi)^{3} f_{\pi}^{4}}\left\{u^{3}+\frac{3}{4 x} \int_{(x-u) / 2}^{(u+x) / 2} d \xi \frac{u^{2}-(2 \xi-x)^{2}}{1+2 \xi^{2}}\right. \\
& \left.\times\left[\left(1+8 \xi^{2}+8 \xi^{4}\right) \arctan \xi-\left(1+4 \xi^{2}\right) \arctan 2 \xi\right]\right\},
\end{aligned}
$$

with the abbreviations $u=k_{f} / m_{\pi}$ and $x=p / m_{\pi}$. Eq.(11) in ref. [3] for the three-body potential from the iterated $1 \pi$-exchange Hartree diagram gets modified to:

$$
\begin{aligned}
U_{3}\left(p, k_{f}\right)= & \frac{6 g_{A}^{4} M m_{\pi}^{4}}{\left(4 \pi f_{\pi}\right)^{4}}\left\{\int _ { y _ { \operatorname { m i n } } } ^ { 1 } d y \left\{\left[2 u x y+\left(u^{2}-x^{2} y^{2}\right) \ln \frac{u+x y}{|u-x y|}\right] \mathcal{A}_{y}\left[\frac{2 s^{2}+s^{4}}{2\left(1+s^{2}\right)}-\ln \left(1+s^{2}\right)\right]\right.\right. \\
& \left.+\int_{x y-s}^{s-x y} d \xi\left[2 u \xi+\left(u^{2}-\xi^{2}\right) \ln \frac{u+\xi}{u-\xi}\right] \frac{(x y+\xi)^{5}}{\left[1+(x y+\xi)^{2}\right]^{2}}\right\} \\
& \left.+\int_{-1}^{1} d y \int_{0}^{u} d \xi \frac{\xi^{2}}{x}\left[\frac{2 \sigma^{2}+\sigma^{4}}{1+\sigma^{2}}-2 \ln \left(1+\sigma^{2}\right)\right] \ln \frac{x+\xi y}{x-\xi y}\right\} .
\end{aligned}
$$

Finally, eq.(13) in ref. 3] for the three-body potential from the iterated $1 \pi$-exchange Fock diagram is replaced by:

$$
\begin{aligned}
U_{3}\left(p, k_{f}\right)= & \frac{3 g_{A}^{4} M m_{\pi}^{4}}{\left(4 \pi f_{\pi}\right)^{4}}\left\{\frac{G^{2}(x)}{8 x^{2}}+\int_{0}^{u} d \xi G(\xi)\left[1+\frac{\xi^{2}-x^{2}-1}{4 x \xi} \ln \frac{1+(x+\xi)^{2}}{1+(x-\xi)^{2}}\right]\right. \\
& +\int_{y_{\min }}^{1} d y \int_{y_{\min }}^{1} d z \frac{\theta\left(y^{2}+z^{2}-1\right)}{4 \sqrt{y^{2}+z^{2}-1}} \mathcal{A}_{y}\left[s^{2}-\ln \left(1+s^{2}\right)\right] \mathcal{A}_{z}\left[\ln \left(1+t^{2}\right)-t^{2}\right] \\
& \left.+\int_{-1}^{1} d y \int_{0}^{u} d \xi \frac{\xi^{2}}{x}\left[\ln \left(1+\sigma^{2}\right)-\sigma^{2}\right]\left(\ln \frac{x+\xi y}{x-\xi y}+\frac{1}{R} \ln \frac{x R+\left(x^{2}-\xi^{2}-1\right) y \xi}{x R+\left(1-x^{2}+\xi^{2}\right) y \xi}\right)\right\},
\end{aligned}
$$


where we have again introduced the auxiliary function:

$$
G(x)=u\left(1+u^{2}+x^{2}\right)-\frac{1}{4 x}\left[1+(u+x)^{2}\right]\left[1+(u-x)^{2}\right] \ln \frac{1+(u+x)^{2}}{1+(u-x)^{2}} .
$$

For the definition of the quantities $y_{\min }, s, \sigma, t$ and $R$ and the antisymmetrization prescription $\mathcal{A}_{y}$ we refer to section 4 .

\section{References}

[1] M. Lutz, B. Friman and Ch. Appel, Phys. Lett. B474 (2000) 7.

[2] N. Kaiser, S. Fritsch and W. Weise, Nucl. Phys. A697 (2002) 255.

[3] N. Kaiser, S. Fritsch and W. Weise, Nucl. Phys. A700 (2002) 343.

[4] A. Bohr and B.R. Mottelson, Nuclear Structure, Vol.I, Benjamin, (1969), chapt. 2.4.

[5] P.E. Hodgson, Growth Points in Nuclear Physics, Vol. 3, Pergamon Press, (1981), chapt. 2.

[6] S. Fritsch, N. Kaiser and W. Weise, Phys. Lett. B545 (2002) 73.

[7] S. Fritsch and N. Kaiser, Eur. Phys. J. A17 (2003) 11.

[8] S. Fritsch and N. Kaiser, Eur. Phys. J. A21 (2004) 117.

[9] J. Kuckei, F. Montani, H. Müther and A. Sedrakian, Nucl. Phys. A723 (2003) 32.

[10] C. Ordonez, L. Ray and U. van Kolck, Phys. Rev. C53 (1996) 2086.

[11] D.R. Entem and R. Machleidt, Phys. Rev. C68 (2003) 041003; and references therein.

[12] E. Epelbaum, W. Glöckle and Ulf-G. Meißner, nucl-th/0405048 and references therein.

[13] N. Kaiser, R. Brockmann and W. Weise, Nucl. Phys. A625 (1997) 758.

[14] N. Kaiser, S. Gerstendörfer and W. Weise, Nucl. Phys. A637 (1998) 395.

[15] M. Ericson and A. Figureau, J. Phys. G7 (1981) 1197.

[16] A. Akmal, V.R. Pandharipande and D.G. Ravenhall, Phys. Rev. C58 (1998) 1804; and references therein.

[17] S.C. Pieper, V.R. Pandharipande, R.B. Wiringa and J. Carlson, Phys. Rev. C64 (2001) 014001; and references therein.

[18] M.M. Pavan et al., Phys. Scr. T87 (2000) 65.

[19] I. Sick, private communication. See also: D.B. Day et al., Phys. Rev. C40 (1989) 1011.

[20] D. Vretenar, G.A. Lalazissis, R. Behnsch, W. Pöschl and P. Ring, Nucl. Phys. A621 (1997) 853.

[21] N.M. Hugenholtz and L. Van Hove, Physica 24 (1958) 363.

[22] C. Mahaux and R. Sartor, Adv. Nucl. Phys. 22 (1991) 1; and references therein.

[23] G.Q. Li, R. Machleidt and R. Brockmann, Phys. Rev. C45 (1992) 2782.

[24] P. Grange, J.P. Cugnon and A. Lejeune, Nucl. Phys. A473 (1987) 365.

[25] P. Bozek and P. Czerski, Eur. Phys. J. A11 (2001) 271; and references therein.

[26] S.K. Bogner, T.T.S. Kuo and A. Schwenk, Phys. Rep. 386 (2003) 1; and references therein.

[27] A. Schwenk, G.E. Brown and B. Friman, Nucl. Phys. A703 (2002) 745.

[28] H. Feldmeier, T. Neff, R. Roth and J. Schnack, Nucl. Phys. A632 (1998) 61.

[29] V.M. Galitskii, Sov. Phys.-JEPT 7 (1958) 104.

[30] J.M. Luttinger, Phys. Rev. 121 (1961) 942.

[31] R.W. Hasse and P. Schuck, Nucl. Phys. A445 (1985) 205. 
[32] M. Bender, P.-H. Heenen and P.-G. Reinhard, Rev. Mod. Phys. 75 (2003) 121; and references therein.

[33] M. Beiner, H. Flocard, N. Van Giai and P. Quentin, Nucl. Phys. A238 (1975) 29.

[34] E. Chabanat, P. Bonche, P. Haensel, J. Meyer and R. Schaeffer, Nucl. Phys. A635 (1998) 231; and references therein.

[35] F. Tondour, S. Goriely, J.M. Pearson and M. Onsi, Phys. Rev. C62 (2000) 024308.

[36] N. Kaiser, S. Fritsch and W. Weise, Nucl. Phys. A724 (2003) 47.

[37] N. Kaiser, Phys. Rev. C68 (2003) 054001.

[38] J.W. Negele and D. Vautherin, Phys. Rev. C5 (1972) 1472.

[39] M. Brack, C. Guet and H.B. Haakansson, Phys. Rep. 123 (1985) 275.

[40] J.P. Blaizot, Phys. Rep. 64 (1980) 171.

[41] J. Dobaczewski, work in progress and private communications.

[42] J.B. Natowitz et al., Phys. Rev. Lett. 89 (2002) 212701.

[43] V.A. Karnaukhov et al., Phys. Rev. C67 (2003) 011601(R).

[44] B. Friedman and V.R. Pandharipande, Nucl. Phys. A361 (1981) 502.

[45] G. Sauer, H. Chandra and U. Mosel, Nucl. Phys. A264 (1976) 221.

[46] J.I. Kapusta, "Finite-Temperature Field Theory", Cambridge University Press, (1989), chapt. 10.

[47] J. Carlson, J. Morales, V.R. Pandharipande, and D.G. Ravenhall, Phys. Rev. C68 (2003) 025802 .

[48] C. Ordonez and U. van Kolck, Phys. Lett. B291 (1992) 459.

[49] D. Vretenar, T. Niksic and P. Ring, Phys. Rev. C68 (2003) 024310.

[50] P.A. Seeger and W.M. Howard, Nucl. Phys. A238 (1975) 491.

[51] R.J. Furnstahl, Nucl. Phys. A706 (2002) 85.

[52] T. Niksic, D. Vretenar, P. Finelli and P. Ring, Phys. Rev. C66 (2002) 024306.

[53] N. Kaiser, Nucl. Phys. A709 (2002) 251.

[54] N. Kaiser, Phys. Rev. C (2004) in print. 